\title{
Nonlinear Dynamics and Chaos in Conformational Changes of Mechanical Metamaterials
}

\author{
Jason Z. Kim® \\ Department of Bioengineering, University of Pennsylvania, Philadelphia, Pennsylvania 19104, USA \\ Zhixin Lu우 \\ Department of Bioengineering, University of Pennsylvania, Philadelphia, Pennsylvania 19104, USA \\ Ann S. Blevins \\ Department of Bioengineering, University of Pennsylvania, Philadelphia, Pennsylvania 19104, USA \\ Dani S. Bassett๑* \\ Department of Bioengineering, University of Pennsylvania, Philadelphia, Pennsylvania 19104, USA, \\ Department of Physics \& Astronomy, University of Pennsylvania, Philadelphia, Pennsylvania 19104, USA, \\ Department of Electrical \& Systems Engineering, University of Pennsylvania, \\ Philadelphia, Pennsylvania 19104, USA, \\ and The Santa Fe Institute, Santa Fe, New Mexico 87501, USA
}

(Received 25 November 2020; revised 17 December 2021; accepted 12 January 2022; published 4 March 2022)

From enzyme binding to robot grasping, the function of many mechanical systems depends upon large, coordinated motions of their components. Such motions arise from a network of physical interactions in the form of links (edges) that transmit forces between constituent elements (nodes) and have been fruitfully modeled in known networks. However, the principled design of precise motions in novel networks is made difficult by the number and nonlinearity of interactions. Here, we formulate a simple but powerful framework for designing fully nonlinear motions using concepts from dynamical systems theory. We demonstrate that a small network unit acts as a one-dimensional map between the distances across pairs of nodes, and we represent the act of combining units as an iteration of this map. By tying the map's attractors and their stability to the shape and folding sequence in a network of combined units, we program the precise coordinated motion between arbitrarily complex macroscopic shapes, the exact folding sequence between the shapes, and exotic network behaviors such as a mechanical AND gate and a period-doubling route to chaos. Further, we construct a unit with a 3-cycle that combines to form a lattice with any positive integer period as a result of Sharkovskii's theorem. Finally, we construct physical networks and analyze the effect of bond elasticity to demonstrate the framework's potential and versatility. The precise design of shape change and folding sequence makes this framework ideal as a starting minimal model for many applications, such as robotics, providing a promising direction for future work in metamaterials.

DOI: $10.1103 /$ PhysRevX.12.011042

\section{INTRODUCTION}

From cell membrane channels [1] to medical stents [2], mechanical systems play crucial roles in the natural [3-5] and engineered [6-8] world. What makes these systems useful is their ability to change their geometry in a

\footnotetext{
*To whom correspondence should be addressed. dsb@seas.upenn.edu

Published by the American Physical Society under the terms of the Creative Commons Attribution 4.0 International license. Further distribution of this work must maintain attribution to the author(s) and the published article's title, journal citation, and DOI.
}

Subject Areas: Mechanics, Metamaterials

Nonlinear Dynamics coordinated way to amplify motion or to dramatically change size. Despite their differences, each of these systems can be represented as a mechanical network, where the rigid edges encode constraints due to physical limbs or forces, and the nodes represent joints or constituent elements. A simple and powerful framework for understanding the relationship between network structure and coordinated motion is structural rigidity theory [9], originating from early and seminal work by Maxwell [10-12]. Here, the difference between the numbers of node coordinates and edges yields the number of coordinated motions.

However, the successful design of coordinated motions depends not only on their existence but also on the timeevolving network geometry for their duration. The specific 
geometry is determined by the edge constraints, just as a robot's limbs constrain its configuration. Several works provide design principles relating edge placement to node motions in small networks [13-17] and to detailed singlenode trajectories or local perturbations in large networks $[18,19]$. Other studies explore lattices in the study of topological mechanics in networks [20-23] and in origami [24-26], along with sequential and branched motions [27-31], and they examine design considerations such as flexible deformation [32], connection topology [33,34], and environmental responsivity [35].

Many of these studies take advantage of the simple yet powerful idea to decompose networks into properties of unit cells and their interactions and to study lattices of and defects in-identical unit cells. In this work, we build upon this idea to design complex unit cell properties that yield exotic and chaotic behaviors in lattices of identical cells. We further extend these ideas to program arbitrary shape changes and folding sequences in networks by designing and combining nonidentical cells. Excitingly, many techniques are being developed to physically construct complex networks [36-38]. As the interest in these systems has grown across many disciplines, it is now timely to develop a general framework for designing specific geometric trajectories in large networks.

Here, we develop such a framework by designing specific properties of shape change in unit cells and their interactions. This paper is organized as follows. In Sec. II, we review the mathematical and numerical foundations that we use to study shape changes. In Sec. III, we formalize principles of how the shape change of a network unit comprising few elements determines the shape change of larger networks comprising many such units. In Sec. IV, we reverse engineer this process to design units that, when combined, yield targeted global shape change. In Sec. V, we explore the design space of these units to further design the folding sequence of the network chains. Using these principles, we finally design exotic and nonlinear functions such as a mechanical AND gate in Sec. VI and chaotic conformational change in Sec. VII, and we construct physical networks in Sec. IX.

\section{MATHEMATICAL FRAMEWORK}

\section{A. Constraint counting}

Coordinated motions arise from the arrangement of physical forces between constituent elements such as tension and compression transmitted through a rigid robot limb, which we model as distance constraints (edges) between point particles (nodes). In two-dimensional space, each node $i$ has two coordinates, $x_{i}$ and $y_{i}$, thereby allowing two motions. Each edge $k$ of length $d_{k}$ between nodes $i$ and $j$ must keep a constant length,

$$
\left(x_{i}-x_{j}\right)^{2}+\left(y_{i}-y_{j}\right)^{2}=d_{k}^{2},
$$

(a) Building blocks node: +2 motions

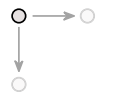

Edge: -1 motion $\leftrightarrow x-0 x \rightarrow$

(b) Combine blocks

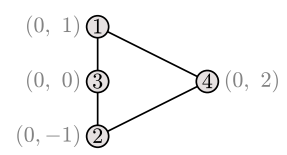

4 nodes: +8 motions 4 edges: -4 motions

4 motions

(c) 3 rigid body motions

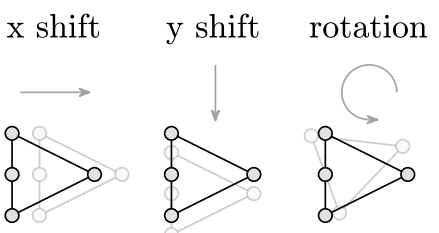

(d) 1 conformational motion
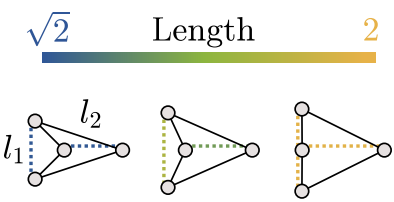

FIG. 1. Constraints and conformational motions. (a) Schematic of a node and an edge embedded in two dimensions, where a node adds two motions (one in each dimension), and an edge removes the one motion that changes its length. (b) Network of four nodes and four edges, yielding a total of four motions, (c) three of which are rigid body motions and (d) one of which is a conformational motion.

thereby removing one motion [Fig. 1(a)]. Hence, the number of motions in a network without redundant constraints (see Sec. II C) is given by

$$
M=2 N-E,
$$

where $N, E$, and $M$ are the numbers of nodes, edges, and motions, respectively. As such, a network of four nodes and four edges contains $8-4=4$ motions [Fig. 1(b)]. Three motions preserve the distance between all nodes through translations and rotation and are called rigid body motions [Fig. 1(c)]. The fourth motion changes the lengths $l_{1}$ and $l_{2}$ between unconnected nodes and is called a conformational motion [Fig. 1(d)].

Throughout, we use the italicized variable $d$ to refer to the distance between nodes connected by an edge, the unitalicized symbol d to refer to the differential operator, and the variable $l$ to refer to the distance between nodes that are not connected by an edge.

\section{B. Defining the set of motions}

While we can visually intuit the motions of networks comprising few nodes as in Fig. 1(d), we seek a quantitative framework to define such motions for much larger networks. We outline a common framework from rigidity theory [21] that relates changes in node coordinates to changes in edge lengths. Then, the set of allowed node motions are those that cause zero change in edge length.

For a set of $N$ nodes $\mathcal{V}=\{1, \ldots, N\}$ connected by $E$ edges $\mathcal{E} \subseteq \mathcal{V} \times \mathcal{V}$, any edge $k$ connecting nodes $i$ and $j$ has length $d_{k}$ according to Eq. (1). To relate changes in node 
coordinates to changes in edge lengths, we take the derivative of Eq. (1) and divide by $2 d_{k}$ to yield

$$
\frac{\left(x_{i}-x_{j}\right)}{d_{k}}\left(\mathrm{~d} x_{i}-\mathrm{d} x_{j}\right)+\frac{\left(y_{i}-y_{j}\right)}{d_{k}}\left(\mathrm{~d} y_{i}-\mathrm{d} y_{j}\right)=\mathrm{d} d_{k} .
$$

We now obtain the desired relationship between node motions, $\mathrm{d} x$ and $\mathrm{d} y$, to changes in edge length, $\mathrm{d} d_{k}$.

Next, we notice that if we know the node positions $x$ and $y$ as constants, then the equation is linear in the node motion variables: $\mathrm{d} x$ and $\mathrm{d} y$. Because of this linearity, if we write $\boldsymbol{x}$ as the $2 N$-dimensional vector of node positions, $\mathrm{d} \boldsymbol{x}$ as the $2 N$-dimensional vector of node motions, and $\mathrm{d} \boldsymbol{d}$ as the $E$-dimensional vector of changes in edge length, then we can concisely write Eq. (3) for all edges as

$$
C \mathrm{~d} \boldsymbol{x}=\mathrm{d} \boldsymbol{d}
$$

Here, $C=C(\boldsymbol{x})$ is the compatibility matrix of size $E \times 2 N$ comprised predominantly of zeros [21]. For every $k$ th row in $C$, the only nonzero entries are $\left(x_{i}-x_{j}\right) / d_{k}$ multiplying $\mathrm{d} x_{i},\left(x_{j}-x_{i}\right) / d_{k}$ multiplying $\mathrm{d} x_{j},\left(y_{i}-y_{j}\right) / d_{k}$ multiplying $\mathrm{d} y_{i}$, and $\left(y_{j}-y_{i}\right) / d_{k}$ multiplying $\mathrm{d} y_{j}$. Hence, every row of Eq. (4) is precisely Eq. (3) for the edge corresponding to that row, and the compatibility matrix maps node motions to bond extensions across the entire network.

Finally, because the edges are rigid, we set $\mathrm{d} \boldsymbol{d}=\mathbf{0}$ such that all infinitesimal node motions that cause zero change in edge length must satisfy

$$
C \mathrm{~d} \boldsymbol{x}=\mathbf{0} .
$$

As a result, the set of all infinitesimal motions that yield zero change in edge length is given by the null space $\mathcal{N}(C)$. In our simple four-node network, the three rigid body motions in Fig. 1(c) and the one conformational motion in Fig. 1(d) are all contained in the null space of $C$. Collectively, these motions are referred to as zero modes.

\section{Constraint-counting revisited}

Through the compatibility matrix, we can make a more nuanced statement about the number of coordinated motions through the Calladine index theorem [21]. We have already seen that the compatibility matrix maps node motions to bond extensions in Eq. (4). Additionally, the equilibrium matrix, $Q=C^{\top}$, maps bond tensions $t$ to node forces $f$ such that

$$
Q \boldsymbol{t}=\boldsymbol{f}
$$

Here, the null space of $Q$ then represents vectors of bond tensions that cancel out to yield zero net force at the nodes and are referred to as states of self-stress (SSS) [21]. These SSS often arise from overconstraining the network through the addition of redundant bonds but can also arise from geometric singularities through kinematic bifurcations [14].

The Calladine index theorem relates the columnspaces and null spaces of $C$ and $Q$. From the rank-nullity theorem, we know that a system with $S$ states of self-stress has the relation

$$
\operatorname{rank}(Q)+S=E,
$$

and that a system embedded in two dimensions with $M$ zero modes has the relation

$$
\operatorname{rank}(C)+M=2 N .
$$

Because $\operatorname{rank}(Q)=\operatorname{rank}(C)$, we substitute to obtain

$$
M=2 N-E+S .
$$

Hence, the number of motions is almost the same as for constraint counting in Eq. (2) while accounting for SSS (see Ref. [21] for additional details). Unless stated otherwise, our systems have $S=0$.

\section{Instantiating and simulating networks}

Now that we have defined the space of allowed node motions, how do we evolve our networks along their conformational motion? Our approach involves four steps, where the ultimate goal is to remove the rigid body motions from the set of all motions to isolate the conformational motion.

First, at simulation step $k=0$, we instantiate our network by choosing the node coordinates $x_{i}[0]$ and $y_{i}[0]$ for $i=1, \ldots, N$, and by defining the edge placements between node pairs. Importantly, we note that choosing the node coordinates and edge placements determines the length $d_{k}$ of each edge $k$. Hence, we are able to fully construct the compatibility matrix $C$ from the node coordinates and edge placements alone.

Second, at simulation step $k$ starting at $k=0$, we collect all of the node positions into a $2 N$-dimensional vector $\boldsymbol{x}[k]$, construct our compatibility matrix $C[k]=C(\boldsymbol{x}[k])$, and compute the set of allowed node motions through the null space $\mathcal{N}(C[k])$. We collect the basis set that spans the null space as a $2 N \times M$ matrix, $P[k]$.

Third, we define the basis set of rigid body motions $R[k]=\left[\boldsymbol{x}_{x}, \boldsymbol{x}_{y}, \boldsymbol{x}_{\text {rot }}[k]\right]$ and quotient them out of our set of allowed motions to yield the conformational motion. Numerically, we can implement this quotient by taking the null space of the projection of $R[k]$ onto $P[k]$ as $\mathcal{N}\left(R[k]^{\top} P[k]\right)$, collecting the basis vectors that span this null space into an $M \times M-3$ matrix $Q$, and projecting the null space back into the coordinate space as

$$
\mathrm{d} \boldsymbol{x}[k]=P[k] Q .
$$


Unless stated otherwise, $\mathrm{d} \boldsymbol{x}[k]$ will always be a vector because our systems will always have $M=4$ motions, such that removing the three rigid body motions will leave behind one conformational motion. We normalize the conformational motion such that $\mathrm{d} \hat{\boldsymbol{x}}[k]=\mathrm{d} \boldsymbol{x}[k] /\|\boldsymbol{x}[k]\|_{2}$.

Finally, we evolve the network forward by numerically integrating the differential in Eq. (10). Specifically, at each time step $k$, we evolve the node positions forward from $\boldsymbol{x}[k]$ to $\boldsymbol{x}[k+1]$ using a tenth order Runge-Kutta scheme that relies on the evaluation of steps 2 and 3 at each substep [39]. The reason for such a high-order integration scheme will become clear in the results, as high accuracy of numerical integration is necessary for simulating networks with chaotic behaviors.

\section{E. Motivating statement and outline}

Conformational motions endow networks with functions that depend on targeted changes in shape. The design of a specific shape change is determined by the node positions

(a) How do we combine many nodes and edges...

$N$ nodes

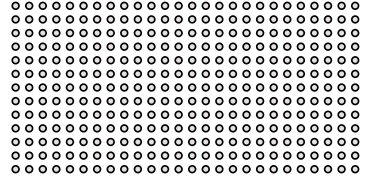

,

(b)

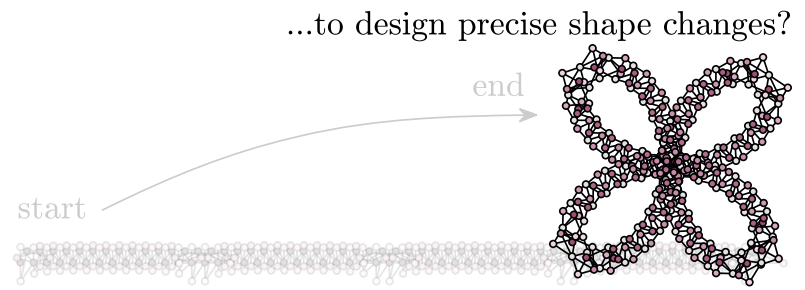

(c)

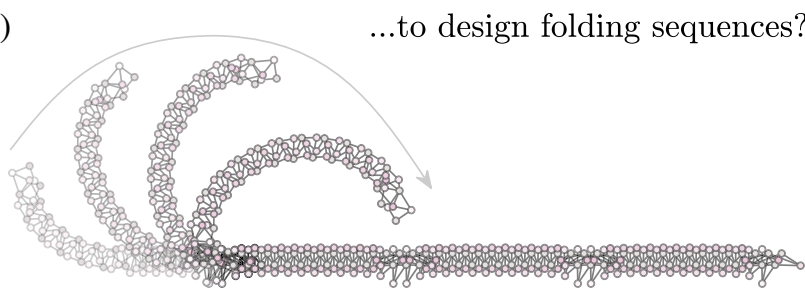

(d)

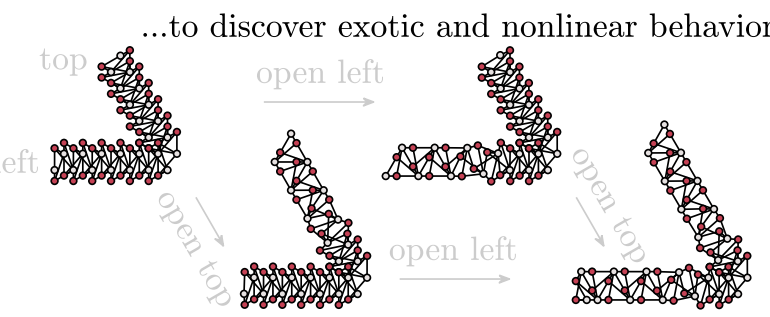

FIG. 2. Motivation for the results. (a) Set of 338 nodes and 672 edges that, through our results, can be designed to have (b) precise shape changes and (c) folding sequences. (d) Network that has been designed to behave as a mechanical AND gate. and edge placements and is made difficult by the nonlinearity of the constraints, even in networks of few nodes [Eq. (1)]. Given the vast design space in systems of many nodes and edges [Fig. 2(a)], what are the organizational principles that enable us to design precise shape changes [Fig. 2(b)], folding sequences [Fig. 2(c)], and exotic and nonlinear behavior [Fig. 2(d)]?

\section{FOUR-BAR LINKAGE EXAMPLE}

\section{A. Motion of one unit acts as a map}

To understand the principles that govern shape change in networks of many elements, we first develop intuition for shape change in units comprising a few elements. Specifically, we study the shape change of the four-bar linkage previously shown in Fig. 1. We observe that the length between unconnected nodes-namely, length $l_{k}$ between nodes 1 and 2, and $l_{k+1}$ between nodes 3 and 4 - change throughout the motion (Fig. 3). If we plot these two lengths along the motion, we obtain a curve that maps length $l_{k}$ to length $l_{k+1}$ as a function

$$
l_{k+1}=f\left(l_{k}\right)
$$

The equation of this specific unit's map is $f\left(l_{k}\right)=$ $\sqrt{5-l_{k}^{2} / 4}-\sqrt{1-l_{k}^{2} / 4}$, and it is derived via the edge constraints given in Eq. (1).

\section{B. Combining units acts as a map iteration}

This map immediately motivates a simple and powerful way to construct a network of many nodes whose shape change is fully known. Specifically, if we could somehow combine these units such that the lengths of subsequent

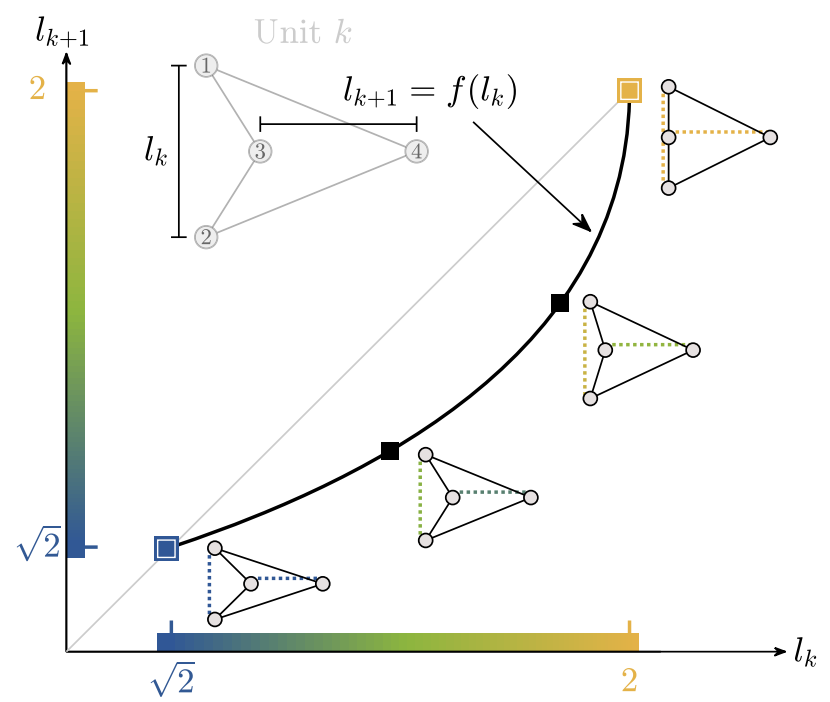

FIG. 3. Conformational motion as a map. Plot of the lengths $l_{k}$ and $l_{k+1}$ between unconnected nodes in the example unit (Fig. 1) as it changes shape. 
units are functions of the lengths of previous units, then we could write the conformation of all units as a function of the first, such that $l_{k+1}=f^{k}\left(l_{1}\right)$. Through this relation, we could simply and explicitly parametrize the shape of the entire network through a single parameter.

To achieve this relationship, consider two four-bar linkage units, 1 and 2. Unit 1 has length $l_{1}$ between nodes 1 and 2 , and length $f\left(l_{1}\right)$ between nodes 3 and 4 . Unit 2 has length $l_{2}$ between nodes 1 and 2 , and length $f\left(l_{2}\right)$ between nodes 3 and 4 (Fig. 4). If we set $l_{2}$ of unit 2 equal to $f\left(l_{1}\right)$ of unit 1 , then we can combine units 1 and 2 by merging nodes, by which we mean overlapping and gluing node 3 of unit 1 and node 1 of unit 2 such that they become the same node, and by overlapping and gluing node 4 of unit 1 and node 2 of unit 2 in the same way. Thus, the lengths of unit 2 are determined by those of unit 1 , such that $f\left(l_{2}\right)=$ $f\left(f\left(l_{1}\right)\right)$ (Fig. 4, top).

Afterwards, we add another unit-unit 3-whose nodes 1 and 2 define length $l_{3}$, and whose nodes 3 and 4 define length $f\left(l_{3}\right)$. By setting $l_{3}$ of unit 3 equal to length $f\left(l_{2}\right)$ of unit 2 , we can combine units 2 and 3 by merging node 3 of unit 2 with node 1 of unit 3 such that they become the same node, and by merging node 4 of unit 2 with node 2 of unit 3 in the same way. Thus, the shape of unit 3 is also determined by that of unit 1 , such that $f\left(l_{3}\right)=f\left(f\left(l_{2}\right)\right)=$ $f\left\{f\left[f\left(l_{1}\right)\right]\right\}$ (Fig. 4, middle).

We can continue this process as often as we like, such that unit $k$ combines with unit $k+1$ by first setting length $l_{k+1}$ of unit $k+1$ equal to $f\left(l_{k}\right)$ of unit $k$, and then merging node 3 of unit $k$ with node 1 of unit $k+1$, and node 4 of
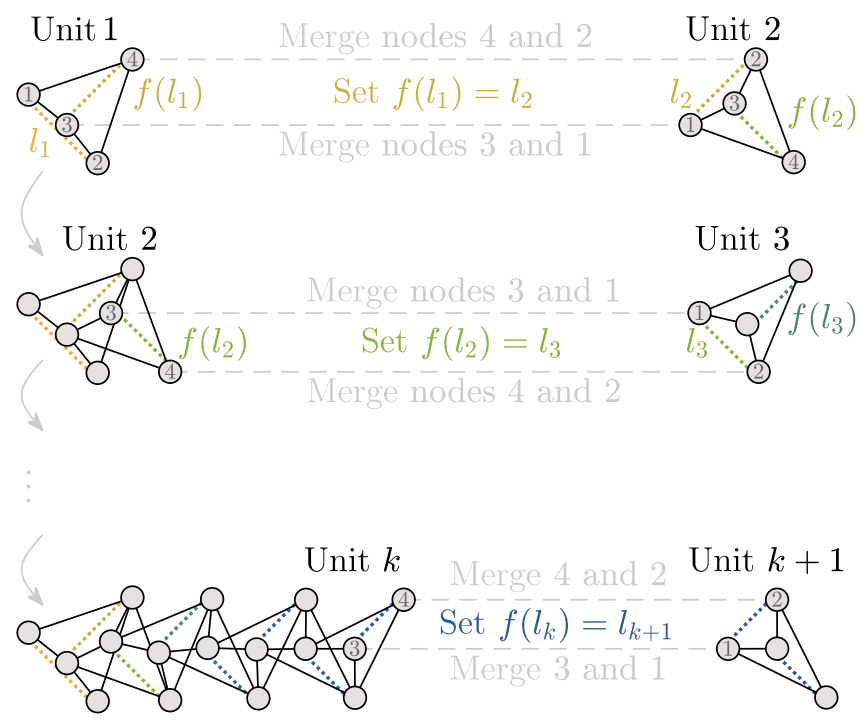

FIG. 4. Combine units by merging nodes. A unit $k+1$ is combined with unit $k$ by merging nodes. First, the length $l_{k+1}$ between nodes 1 and 2 of unit $k+1$ is set equal to the length $f\left(l_{k}\right)$ between nodes 3 and 4 of unit $k$. Then, node 2 of unit $k+1$ is merged to node 4 of unit $k$ by overlapping and gluing the nodes such that they become the same node, and node 1 of unit $k+1$ is merged to node 3 of unit $k$ in the same manner. unit $k$ with node 2 of unit $k+1$ (see Sec. XII of the Supplemental Material [40] for an alternate formulation). In this way, the shape of unit $k+1$ is determined by that of unit 1 , such that

$$
l_{k+1}=f^{k}\left(l_{1}\right),
$$

as we show in the bottom of Fig. 4. This equation is referred to as an iterated map in nonlinear dynamics.

\section{Visualizing map iteration as a cobweb plot}

To develop an intuition for the relationship between the map iteration and the network chain's geometry, we can visually represent the map iteration as a cobweb plot [41], which consists of horizontal and vertical lines in the plot of $l_{k+1}=f\left(l_{k}\right)$. Such visualizations will show us the properties of the map [Eq. (12)] that are useful to design.

To draw a cobweb plot, we start by drawing the map between lengths $l_{k+1}=f\left(l_{k}\right)$ [Fig. 5(a)]. A cobweb plot begins at the initial length $l_{1}$ along the horizontal axis, and we draw a vertical line up to the function $f$ until it reaches the ordered pair $\left(l_{1}, f\left(l_{1}\right)\right)$. In our example, one of the cobweb plots begins at $l_{1}=1.95$ and has a vertical line drawn to the ordered pair $(1.95, f(1.95))$. Next, a horizontal line is drawn to the line $l_{k+1}=l_{k}$ to reach the ordered pair $\left(f\left(l_{1}\right), f\left(l_{1}\right)\right)$ to prepare the coordinates for the next function evaluation. This process of drawing a vertical line to ordered pair $\left(l_{k}, f\left(l_{k}\right)\right)$, followed by a horizontal line to the diagonal $l_{k}=l_{k+1}$, is repeated for as many units as are in the network chain.

As a result, each ordered pair $\left(l_{k}, f\left(l_{k}\right)\right)$ represents the conformation of unit $k$, and the entire cobweb plot represents the conformation of the entire network chain at a particular initial length $l_{1}$. In Fig. 5(a), we show three cobweb plots corresponding to three network chains, where $l_{1}$ of unit 1 begins at $l_{1}=2, l_{1}=1.95$, and $l_{1}=$ $\sqrt{2}$ [Fig. 5(a)], with the corresponding network conformations shown in Figs. 5(b)-5(d), respectively. Importantly, we note that these three networks are identical in terms of bond lengths and connectivity. They only differ in the initial length $l_{1}$. Additionally, we note that the network can continuously deform its geometry from Fig. 5(b) to Fig. 5(d) along one conformational motion, without changing bond lengths or connection topology.

We highlight two key observations from these cobweb plots. The first is that there are some points where all units are identical, namely, $l=\sqrt{2}$ and $l=2$ [Figs. 5(b) and 5(d)]. This property is, in some sense, ideal because we can simply and precisely know the conformation of every unit in the network. The second observation is that if the initial length is in between these points such that $\sqrt{2}<l_{1}<2$, then the units seem to converge to $\sqrt{2}$ [Fig. 5(c)]. This property clues us in to how we can design the folding sequence of the network. 
(a) Each network drawn on a cobweb plot

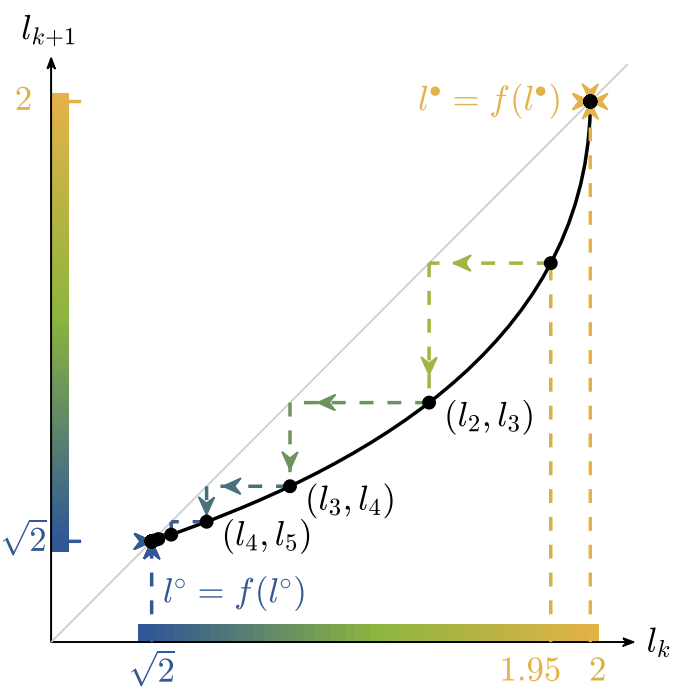

(b)

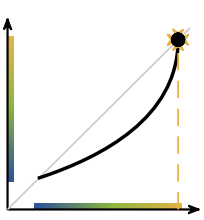

Fixed point: every unit repeats because $l^{\bullet}=f\left(l^{\bullet}\right)$

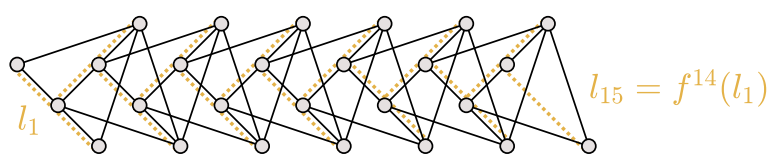

(c)

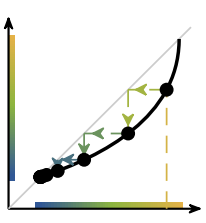

(d)

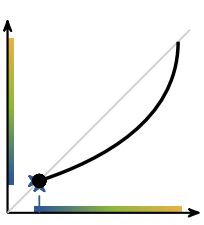

Units move from unstable fixed points to stable ones

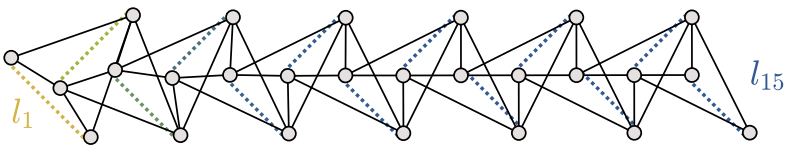

Fixed point: every unit repeats because $l^{\circ}=f\left(l^{\circ}\right)$

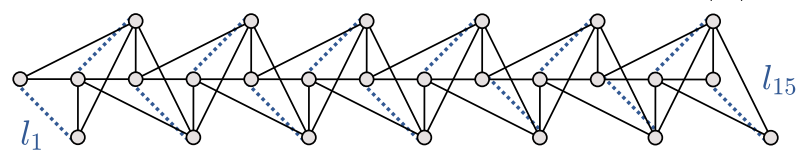

FIG. 5. Shape and folding sequence of iterated maps. (a) Plot of the curve $l_{k+1}=f\left(l_{k}\right)$ for the four-bar linkage example, with three cobweb plots drawn at initial lengths of $l_{1}=2,1.95$, and $\sqrt{2}$. In diagram (b), we show that, at an initial length of $l_{1}=2$, all subsequent units $k$ also have the identical shape $l_{k}=2$ because $f(2)=2$. In diagram (c), at an initial length of $\sqrt{2}<l_{1}=1.95<2$, the subsequent units are no longer identical, and they converge towards the stable fixed point at $l^{\circ}=\sqrt{2}$. In diagram (d), at an initial length of $l_{1}=\sqrt{2}$, all subsequent units $k$ also have the identical shape $l_{k}=\sqrt{2}$.

\section{Network conformation known at fixed points}

While it is true that the conformation of every unit $k$ is determined by Eq. (12), the continued analytical or numerical evaluation of the map $f$ to determine length $l_{k+1}=f^{k}\left(l_{1}\right)$ is quite cumbersome. However, there are special lengths $l^{*}$ known as fixed points that map back to themselves such that

$$
l^{*}=f\left(l^{*}\right)
$$

where the conformation of every unit is easily known. This is because if $f\left(l^{*}\right)=l^{*}$, then $f^{k}\left(l^{*}\right)=l^{*}$, and every unit is in the same conformation.

In our four-bar linkage example, these fixed points are significant because every unit takes on an identical, repeating conformation, which we will refer to as a periodic state. We show the network in the $l^{\circ}=2$ periodic state in Fig. 5(b) and in the $l^{\circ}=\sqrt{2}$ periodic state in Fig. 5(d). In between these two states is an intermediary conformation when $\sqrt{2}<l_{1}<2$, as shown in Fig. 5(c). Here, the network is still constructed from the same four-bar linkage unit with the same bond lengths and connection topology as the previous and subsequent units. We refer to this construction as one having a periodic structure. However, because the length of any unit $k$ does not repeat across the network, it does not have a periodic state.

The motivation and significance for studying these fixed points is that at the fixed points, the conformation of every unit is simply and completely known. Hence, in Sec. IV, we fulfill our first aim, to design precise shape changes in networks of many elements [Fig. 2(b)], by designing units that adopt precise geometries at a common fixed point.

\section{E. Folding sequence determined by stability}

In addition to the conformation of the network chain, the iterated map can also tell us about the change in conformation, or the folding sequence, of the network. This change is simply understood by taking the derivative of Eq. (11) to yield the slope

$$
s_{k}=\frac{\mathrm{d} l_{k+1}}{\mathrm{~d} l_{k}}=f^{\prime}\left(l_{k}\right) .
$$

Intuitively, for any unit $k$, the slope simply tells us whether a perturbation in length $l_{k}$ yields a larger or smaller perturbation in length $l_{k+1}$. If the slope $\left|s_{k}\right|<1$, then the magnitude of perturbation decreases, and the map at the point $l_{k}$ is said to be stable. If the slope $\left|s_{k}\right|>1$, then the magnitude of perturbation increases, and the map at $l_{k}$ is said to be unstable. If $\left|s_{k}\right|=1$, the magnitude of perturbation remains the same, and the map is said to be marginally stable. When considering this same change in the iterated map equation for the entire network chain, we obtain

$$
s=\frac{\mathrm{d} l_{k+1}}{\mathrm{~d} l_{1}}=\prod_{i=1}^{k+1} s_{k},
$$

which tells us whether a perturbation in $l_{1}$ will be larger or smaller than a perturbation in $l_{k+1}$. 
If $|s|>1$, the perturbation in $l_{k+1}$ will be larger than that at $l_{1}$, and the network will begin changing conformation at the $l_{k+1}$ end. In our specific example, the fixed point $l^{\bullet}=2$ is unstable because the slope of the map has magnitude greater than 1 . Hence, in Eq. (15), we observe that $\left|\mathrm{d} l_{k+1}\right|>\mathrm{d} l_{1}$, and we expect the network at the fixed point $l^{*}=2$ in Fig. 5(b) to begin folding from the $l_{k+1}$ end, which is true [Fig. 5(c)].

If $|s|<1$, the perturbation in $l_{k+1}$ will be smaller than that in $l_{1}$, and the network will begin changing conformation at the $l_{1}$ end. In our specific example, the fixed point $l^{\circ}=\sqrt{2}$ is stable because the slope of the map has magnitude less than 1. Hence, in Eq. (15), we observe that $\left|\mathrm{d} l_{k+1}\right|<\mathrm{d} l_{1}$, and we expect the network at the fixed point $l^{\circ}=\sqrt{2}$ in Fig. 5(d) to begin folding from the $l_{1}$ end, which is true [Fig. 5(c)].

The motivation and significance of studying the stability is that the folding sequence of the network is determined by the stability of the unit maps. Hence, in Sec. V, we fulfill our second aim, to design folding sequences [Fig. 2(c)], by designing units that are stable or unstable at a particular geometry.

\section{DESIGNING NETWORK SHAPE}

Using the fact that we know the conformation of all units when at a fixed point, we seek to achieve our first aim: to design the shape of the network chain when the units are at the fixed points [Fig. 2(b)]. Recalling our previous four-bar linkage example, we immediately encounter a problem: Identical units have identical geometries at fixed points, such that the network chain forms a straight line [Figs. 5(b) and 5(d)]. Thus, we are motivated to design our own, nonidentical units that share fixed points but differ in their precise geometry.

\section{A. Motivating the unit design procedure}

To achieve desired shape changes in the network chain, we require that the composite units satisfy three key properties. We approach these properties in a constructive manner, beginning with a unit comprising a set of three nodes - 1,2 , and 3 - and defining length $l_{k}$ between nodes 1 and 2, length $l_{k+1}$ between nodes 2 and 3, and length $c_{k}$ between nodes 1 and 3 (Fig. 6). We begin with three nodes because that is the smallest number of nodes whereby we can define three independent lengths, $l_{k}, l_{k+1}$, and $c_{k}$, to achieve three desired properties of unit design that yield desired shape changes. We describe these three properties one at a time.

The first property is that, among this set of nodes, there exist two conformations (node coordinate positions) where the lengths between unconnected nodes, $l_{k}$ and $l_{k+1}$, are equal. We refer to the length at the first such conformation as the start fixed point, $l^{\circ}$, and the length at the second such conformation as the end fixed point, $l^{\circ}$ [Fig. 6(a)]. This

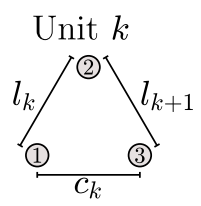

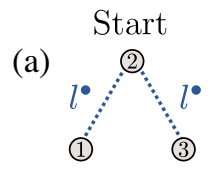

(b)

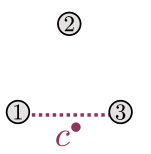

(c)

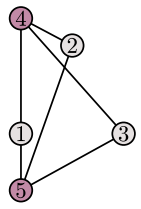

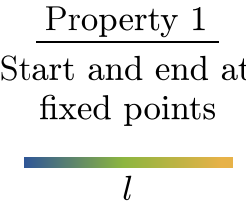

Property 2

Design unit shape through $c$

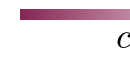

$c$

Property 3
Move through one
conformational
motion
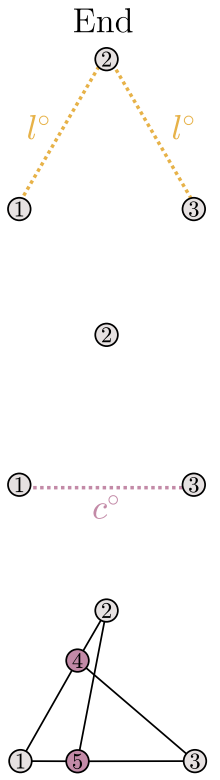

FIG. 6. Properties of unit design. We show a drawing of a unit with three nodes, 1,2 , and 3 , with length $l_{k}$ between nodes 1 and 2 , length $l_{k+1}$ between nodes 2 and 3 , and length $c_{k}$ between nodes 1 and 3 . We seek to design units (a) that start and end at fixed points where $l_{k}=l_{k+1}=l^{\bullet}$ and $l_{k}=l_{k+1}=l^{\circ}$ are valid conformations, (b) whose start and end geometry can be programmed to start at $c^{\bullet}$ and end at $c^{\circ}$, and (c) that can transition from the start to the end shape with one conformational motion.

property ensures that, even if we construct and combine nonidentical units, they will all share identical start and end fixed points. This sharing means that if one unit is in a conformation where a length is at a fixed point such that $l_{k}=l_{k+1}=l^{\circ}$ or $l_{k}=l_{k+1}=l^{\circ}$, then all units, despite being nonidentical, also exist in a conformation where their lengths are at $l^{*}$ or $l^{\circ}$, respectively. Importantly, we note that because fixed points require the lengths $l_{k}$ and $l_{k+1}$ to be identical such that $l_{k}=l_{k+1}$, the three nodes will form an isosceles triangle at the start coordinates (with isosceles side $l^{\circ}$ ) and end coordinates (with isosceles side $l^{\circ}$ ).

The second property differentiates our designed unit from the example four-bar linkage unit, such that we want a design parameter that changes the shape of the unit, and thereby the network, at the fixed points. Hence, the second property is that we have a parameter $c$ through which we can design the shape of the unit at the start fixed point as $c^{\circ}$ and at the end fixed point as $c^{\circ}$ [Fig. 6(b)].

The third and final property is that the unit achieves properties 1 and 2 through one conformational motion. This property allows us to write the lengths between unconnected nodes of a unit as a map [Eq. (11)], thereby 
(a) Unit $k$

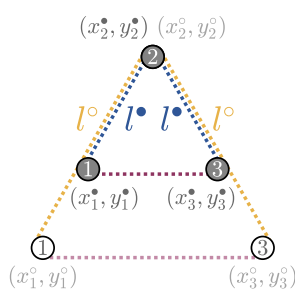

(d) Unit $k$

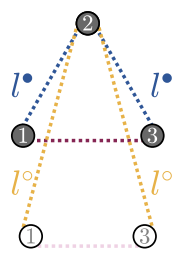

(b) Add extra nodes

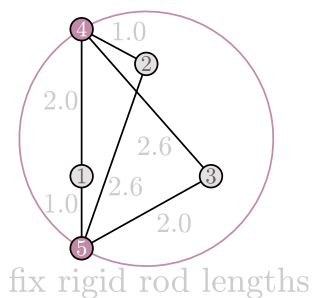

(e) Add extra nodes

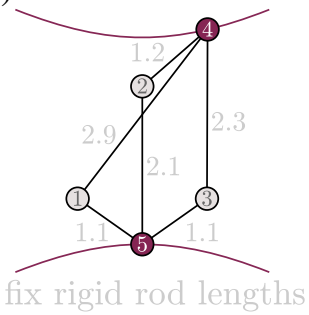

(c) End position

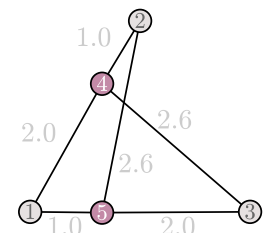

(f) End position

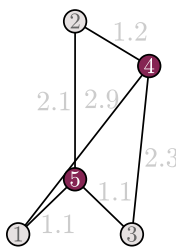

(g)

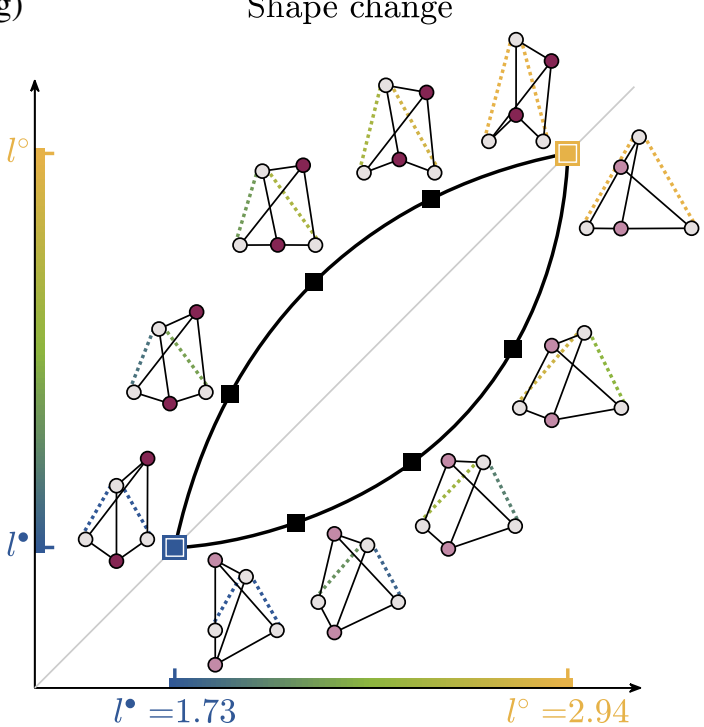

FIG. 7. Designing unit geometry at fixed points. (a) Node coordinates that start at a fixed point $l^{\bullet}$ and end at another fixed point $l^{\circ}$, where the length $c$ is larger at the end than at the start such that $c^{\circ}>c^{\bullet}$. In (b), the maroon curve is the solution space. By placing extra nodes 4 and 5 on the solution space and fully connecting them to nodes 1, 2, and 3 with rigid bonds, (c) there exists an end position of nodes 4 and 5 that keeps all bond lengths the same. (d) Another unit with the same start fixed point $l^{\bullet}$ and end fixed point $l^{\circ}$, but with the length $c$ chosen to be smaller at the end than at the start such that $c^{\circ}<c^{\bullet}$. (e) By placing extra nodes 4 and 5 on the solution space and connecting them to nodes 1,2 , and 3 with rigid edges, (f) there exists an end position that retains all bond lengths. (g) Plots of the conformational motion of both units as they change shape from the start fixed point to the end fixed point.

allowing us to write the lengths across the entire network as an iteration of this map, precisely as in Fig. 4. Because adding edges between nodes 1, 2, and 3 would interfere with desired properties 1 and 2, we constrain our unit to have one conformational motion by adding additional nodes 4 and 5 that are fully connected to nodes 1, 2, and 3 [Fig. 6(c)]. The resulting network will have five nodes corresponding to ten state variables, and six edges corresponding to six constraints, leaving us with $10-6=4$ total motions, and thereby three rigid body motions and one conformational motion.

\section{B. Unit design procedure}

Now that we have motivated the desired properties of our unit, we achieve these properties using a method from prior work [14]. We notice that our base unit forms a triangle with nodes 1,2 , and 3 as the vertices, and that properties 1 and 2 specify the lengths of the triangle edges at the start and end conformations (Fig. 6). By specifying the lengths of all triangle edges, we also specify all node coordinates up to isometric transformations (i.e., translation, rotation, mirror images). Hence, in our unit, designing a unit whose lengths between node pairs are fixed at the start and end conformation is equivalent to designing a unit whose node coordinates are fixed at the start and end conformation.

Hence, we begin our unit design procedure by defining $\left(x_{i}^{\bullet}, y_{i}^{\bullet}\right)$ as the start coordinate of node $i$ and by defining $\left(x_{i}^{\circ}, y_{i}^{\circ}\right)$ as the end coordinate of node $i$ [Fig. 7(a)]. First, we use trigonometry to convert the start edge lengths, $l^{\bullet}, l^{\bullet}, c^{\bullet}$, into start node coordinates, $\left(x_{1}^{\bullet}, y_{1}^{\bullet}\right),\left(x_{2}^{\bullet}, y_{2}^{\bullet}\right),\left(x_{3}^{\bullet}, y_{3}^{\bullet}\right)$, and the end edge lengths, $l^{\circ}, l^{\circ}, c^{\circ}$, into end node coordinates, $\left(x_{1}^{\circ}, y_{1}^{\circ}\right),\left(x_{2}^{\circ}, y_{2}^{\circ}\right),\left(x_{3}^{\circ}, y_{3}^{\circ}\right)$. In this particular example, we choose $l^{\bullet}=\sqrt{3}$ to be the length of the start fixed point, and $l^{\circ}=1.7 \times \sqrt{3}$ to be the length of the end fixed point. We also choose $c^{\bullet}=\sqrt{3}$ to be the start shape parameter and vary the end shape parameter $c^{\circ}$ across units to change the units' shape. These specific lengths were chosen for the purpose of demonstration, and the method does not require these particular lengths.

Now that we have chosen the coordinates for nodes $i \in$ $\{1,2,3\}$ at the start configuration $\left(x_{i}^{\bullet}, y_{i}^{\bullet}\right)$ and end configuration $\left(x_{i}^{\circ}, y_{i}^{\circ}\right)$ that satisfy properties 1 and 2 , we must decide on the position of the two extra nodes $j \in\{4,5\}$ while ensuring that the edges have the same lengths at the start and end configurations. Otherwise, the edges cannot be rigid. This condition is enforced by first setting the squared length of the edges at the start coordinates equal to the squared length of the edges at the end coordinates as

$\left(x_{i}^{\bullet}-x_{j}^{\bullet}\right)^{2}+\left(y_{i}^{\bullet}-y_{j}^{\bullet}\right)^{2}=\left(x_{i}^{\circ}-x_{j}^{\circ}\right)^{2}+\left(y_{i}^{\circ}-y_{j}^{\circ}\right)^{2}$,

where $\left(x_{i}^{\circ}, y_{i}^{\circ}\right)$ and $\left(x_{i}^{\circ}, y_{i}^{\circ}\right)$ are fixed constants [Figs. 8(a) and 8(d)]; we then solve for the start and end positions of the extra nodes, $\left(x_{j}^{\circ}, y_{j}^{\circ}\right)$ and $\left(x_{j}^{\circ}, y_{j}^{\circ}\right)$. The solutions of $\left(x_{j}^{\bullet}, y_{j}^{\bullet}\right)$ and $\left(x_{j}^{\circ}, y_{j}^{\circ}\right)$ for Eq. (16) then define the start and end positions of the added node $j$ that do not change the 


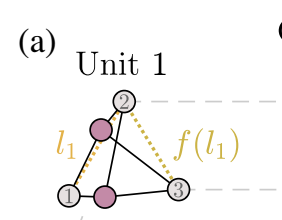

Combine units by merging nodes
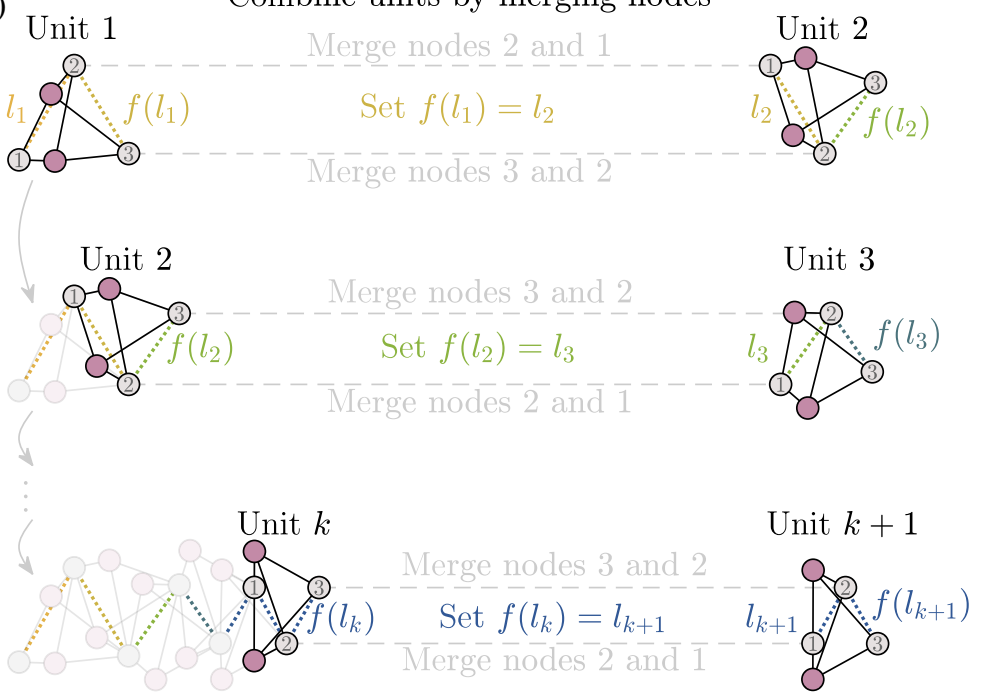

(b)

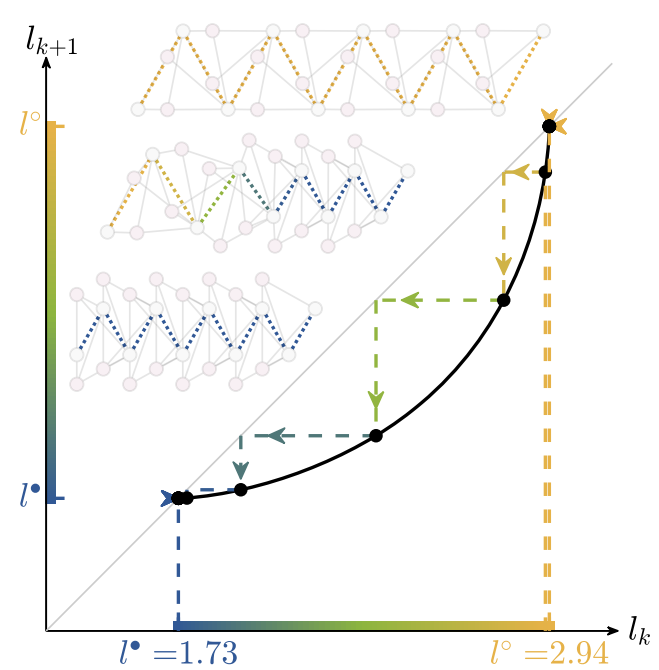

FIG. 8. Representing the combining of designed units as an iterated map. (a) Combining units by merging nodes. A unit $k+1$ is combined with unit $k$ by merging nodes. First, the length $l_{k+1}$ between nodes 1 and 2 of unit $k+1$ is set equal to length $f\left(l_{k}\right)$ between nodes 2 and 3 of unit $k$. Then, node 2 of unit $k+1$ is overlapped and glued together with node 3 of unit $k$ to become a single node, and node 1 of unit $k+1$ is overlapped and glued together with node 2 of unit $k$. (b) Cobweb plot of the curve $l_{k+1}=f\left(l_{k}\right)$ for the designed unit, with three cobweb plots drawn at initial lengths of $l_{1} \approx 1.73,2.93$, and 2.94 , with a drawing of the network corresponding to each cobweb plot.

length of any edges, allowing them to be rigid (see Sec. II of Ref. [40] for details on solving the equations, and Sec. VIII of Ref. [40] for how the node placement fixes the rod lengths).

The solutions to Eq. (16) have a very particular structure. At the start position, it feels intuitive that we should be able to place our extra node $j$ at any location $\left(x_{j}^{*}, y_{j}^{*}\right)$ and connect it with edges of length $d_{k}$ that are equal to the distances between nodes $i$ and $j$. However, when we then move nodes $i \in\{1,2,3\}$ to their desired end position $\left(x_{i}^{\circ}, y_{i}^{\circ}\right)$, we find that there are typically no end positions for the extra node $\left(x_{j}^{\circ}, y_{j}^{\circ}\right)$ that keep the edge lengths the same at $d_{k}$. Intuitively, this lack of solution arises from the fact that while there are three added edges from nodes $i \in$ $\{1,2,3\}$ to node $j$ [and thereby three constraints to satisfy from Eq. (16)], there are only four variables for the extra node positions $\left(x_{j}^{\circ}, y_{j}^{\bullet}, x_{j}^{\circ}, y_{j}^{\circ}\right)$. Hence, there is typically only a one-dimensional solution for the extra node positions, which is defined by a conic section due to the constraints being quadratic [14]. We call the set of start positions $\left(x_{j}^{*}, y_{j}^{*}\right)$ satisfying Eq. (16) the solution space [Figs. 7(b) and 7(e)]. By placing our two nodes $j \in\{4,5\}$ on the solution space [Fig. 7(b)], our unit reaches the desired final position [Fig. 7(c)] and does so along one conformational motion [Fig. $7(\mathrm{~g})]$.

This procedure can now be used to design nonidentical units with the same fixed points $l^{\circ}$ and $l^{\circ}$ but a different end geometry given by the shape parameter $c^{\circ}$. In contrast to the first unit that we designed where the end shape parameter was larger than the start shape parameter
[ $c^{\circ}>c^{\circ}$, Figs. 7(a)-7(c)], we can design a second unit where the end shape parameter is smaller than the start shape parameter $\left[c^{\circ}<c^{\circ}\right.$, Figs. 7(d)-7(f)]. Because the two examples have different end positions, solving Eq. (16) yields different solution spaces [Fig. 7(b) vs Fig. 7(d)]. By adding nodes $j \in\{4,5\}$ on the solution space for the first unit [Fig. 7(b)], the unit has the same bond lengths between the first start and end positions [Fig. 7(c)]. If we place extra nodes $j \in\{4,5\}$ on the solution space for the second unit [Fig. 7(d)], then the unit has the same bond lengths between the second start and end positions [Fig. 7(f)].

This method is not specific to the two examples shown in Fig. 7. For any start $\left(x_{i}^{\circ}, y_{i}^{\circ}\right)$ and end $\left(x_{i}^{\circ}, y_{i}^{\circ}\right)$ positions, we can solve Eq. (16) for the solution space, place extra nodes $j \in\{4,5\}$ along the solution space, connect all nodes $j$ to all nodes $i$, and guarantee that the bond lengths at the start and end positions are equal. The solution space has two important implications. First, if we place the extra nodes $j$ outside of the solution space, then we are guaranteed that our nodes will not go from the start positions $\left(x_{i}^{*}, y_{i}^{*}\right)$ to the end positions $\left(x_{i}^{\circ}, y_{i}^{\circ}\right)$. This is because Eq. (16) defines all placements of node $j$ that preserve edge length. By placing node $j$ outside of the solution space, we are guaranteed to fail at finding an end position of node $j$ that maintains edge length. The second implication is that different desired start and end positions for nodes $i$ define different solution spaces. Between examples 1 and 2, the different end positions $\left(x_{i}^{\circ}, y_{i}^{\circ}\right)$ generate different solution spaces [Fig. 7(b) vs Fig. 7(e)]. This is because the constant parameters of Eq. (16) changed, thereby changing the 
form of the solution space. Varying $c^{\circ}$ generates a range of solution spaces from which we construct our units.

\section{Combining designed units with map iteration}

Now that we have designed units that move from a desired start fixed point $l^{\circ}$ to a desired end fixed point $l^{\circ}$ (property 1) and from a desired start shape $c^{\bullet}$ to a desired end shape $c^{\circ}$ (property 2) along one conformational motion (property 3 ), we need to test whether we can construct network chains as in Fig. 5. To do so, we take our first designed unit from Fig. 7(b) and combine many such units in a manner similar to our four-bar linkage example, whereby we write the conformation of the $k$ th unit as repeated functions of the starting length $l_{1}$.

As in the four-bar linkage example in Sec. III B, we combine units by merging nodes. We begin with our designed unit 1 , with length $l_{1}$ between nodes 1 and 2 , and length $f\left(l_{1}\right)$ between nodes 2 and 3 [Fig. 8(a)]. We then take a second unit with length $l_{2}$ between nodes 1 and 2 , and length $f\left(l_{2}\right)$ between nodes 2 and 3 , and set $l_{2}=f\left(l_{1}\right)$. Finally, we combine units 1 and 2 by merging node 2 of unit 1 with node 1 of unit 2 , and by merging node 3 of unit 1 with node 2 of unit 2. In Fig. 8(a), the nodes to be merged are marked with dashed gray lines. By "merge," we again mean that we move unit 2 over to unit 1 and overlap the nodes to be merged (e.g., node 2 of unit 1 has the same spatial coordinates as node 1 of unit 2) and glue them together such that the overlapped nodes become the same node. In this manner, we can write the conformation of unit 2 as given by $f\left(l_{2}\right)$ as a function of $l_{1}$ through $f\left(l_{2}\right)=f\left(f\left(l_{1}\right)\right)$.

To continue the process of combining units, we add another unit, unit 3 , with length $l_{3}$ between nodes 1 and 2 , and length $f\left(l_{3}\right)$ between nodes 2 and 3 . As before, we set length $l_{3}$ of unit 3 equal to length $f\left(l_{2}\right)$ of unit 2 , and combine units 2 and 3 by merging node 2 of unit 2 with node 1 of unit 3 , and node 3 of unit 2 with node 2 of unit 3 [Fig. 8(a)]. In this manner, we can write the conformation of unit 3 as given by $f\left(l_{3}\right)$ as a function of $l_{1}$ through $f\left(l_{3}\right)=f\left(f\left(l_{2}\right)\right)=f\left(f\left(f\left(l_{1}\right)\right)\right)$.

To continue the process of combining units more generally, we add unit $k+1$ with length $l_{k+1}$ between nodes 1 and 2, and length $f\left(l_{k+1}\right)$ between nodes 2 and 3 . As before, we set length $l_{k+1}$ of unit $k+1$ equal to length $f\left(l_{k}\right)$ of unit $k$, and combine units $k$ and $k+1$ by merging node 2 of unit $k$ with node 1 of unit $k+1$, and by merging node 3 of unit $k$ with node 2 of unit $k+1$ [Fig. 8(a), see Sec. IX of Ref. [40] for extra details on how units are combined]. In this manner, we can write the conformation of unit $k+1$ as given by $f\left(l_{k+1}\right)$ as a function of $l_{1}$ through the iterated map $f\left(l_{k+1}\right)=f^{k}\left(l_{1}\right)$ [Eq. (12)].

Hence, all of the intuitions that we derived regarding the four-bar linkage units in Sec. III translate directly to our own designed units. Specifically, the intuitions that units can be combined such that the shape of unit $k+1$ can be written as the iterated map $l_{k+1}=f\left(l_{k}\right)$ in Sec. III B, that the network's conformational change can be visualized according to a cobweb plot in Sec. III C, that units have identical states at fixed points in Sec. III D, and that the network's folding sequence is determined by the stability of the units in Sec. III E, all translate directly to our designed units (Fig. 8).

\section{Motivating the network design procedure}

Now that we can design general shape changes in units, how can we select the specific units that will yield networks with desired global shape? For example, how do we design nonidentical units that combine to form a network chain that folds into a complex shape such as a quadrifolium [Fig. 9(a)]? To reverse engineer this process, we can reverse the order of this question to ask the following: How can we decompose a desired global shape into a network comprising specific unit shapes?

To answer this question, first recall from Sec. III D that at a fixed point, all unit geometries are known because the lengths $l_{k}$ and $f\left(l_{k}\right)$ are equal to either the start fixed point $l^{\circ}$ or the end fixed point $l^{\circ}$. Second, recall from Sec. IV B that we can choose the start and end values of the shape parameter $-c^{\bullet}$ and $c^{\circ}$, respectively-at the start and end fixed points (Fig. 7). By choosing units whose shape parameters at the end fixed point follow along a trace [Fig. 9(a)], we can design the global shape of the network chain.

\section{E. Selecting units that yield global network shape}

Hence, we seek the end node positions of all units to trace our desired shape at the end fixed point, which we accomplish by tessellating the end global shape with the end node positions $\left(x_{i}^{\circ}, y_{i}^{\circ}\right)$ while enforcing that these positions are at the end fixed point $l^{\circ}$. To demonstrate this process, we construct a network that folds into a quadrifolium as the desired final shape [Fig. 9(a), black]. The specific equation of the trace is given by

$$
r=a \sin (2 \theta), \quad 0 \leq \theta<2 \pi,
$$

where $r$ and $\theta$ represent the radial and angular coordinates of the curve, and we use $a=16.2$ in our example.

To convert this curve into a network, we tessellate the curve with node coordinates $\left(x_{i}^{\circ}, y_{i}^{\circ}\right)$ [Fig. 9(a)]. In this figure, each triangle corresponds to the final shape of one unit, where the corners are the final node positions $\left(x_{i}^{\circ}, y_{i}^{\circ}\right)$, the isosceles sides are $l_{k}=l_{k+1}=l^{\circ}$ (gold), and the nonisosceles side is the shape variable $c^{\circ}$ (purple). The reason why each unit forms an isosceles triangle with isosceles edges between nodes 1 and 2, and between nodes 2 and 3, is because, at a fixed point, the length $l_{k}$ between nodes 1 and 2 and the length $l_{k+1}$ between nodes 2 and 3 are equal to the fixed point length $l_{k}=l_{k+1}=l^{\circ}$. The reason why the units are merged along the isosceles edge is because the units combine by merging nodes that define $l_{k}$ and $l_{k+1}$, which, at the fixed point, is the isosceles edge. 
(a) Tesselate curve with isosceles triangles
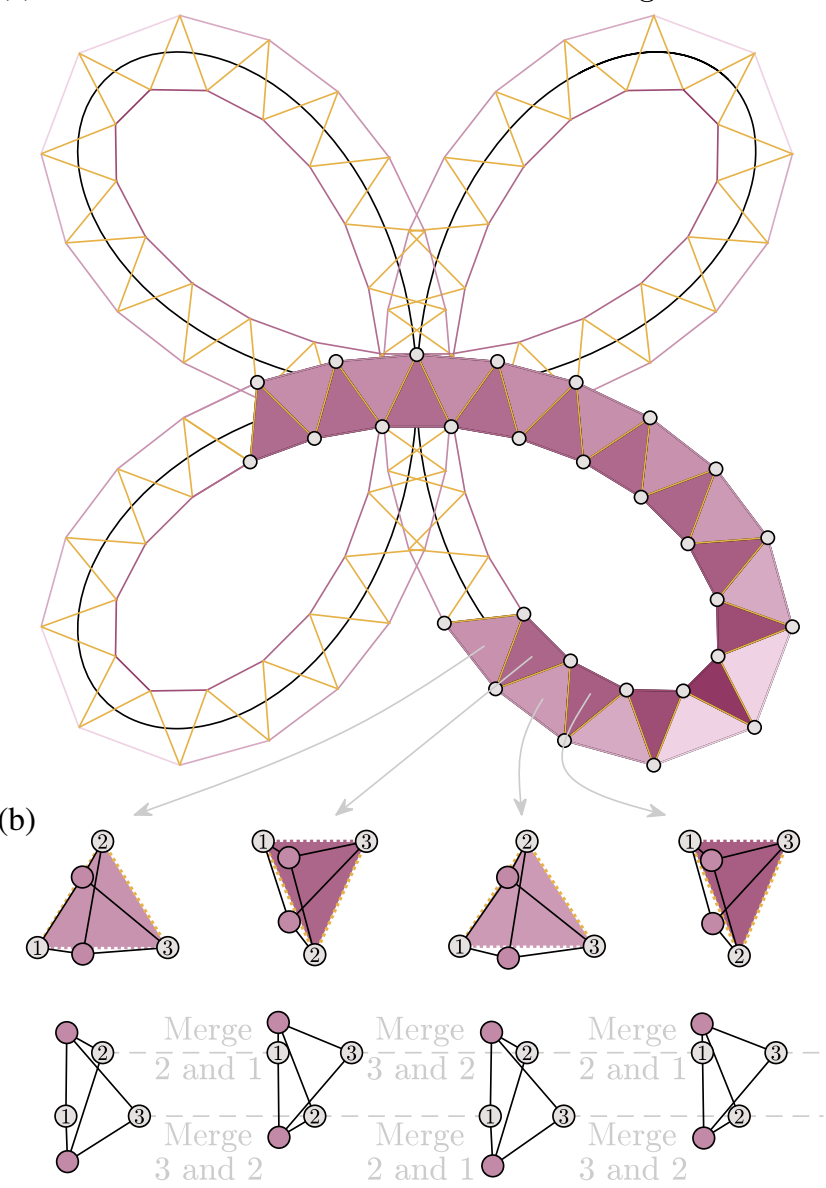

(c)

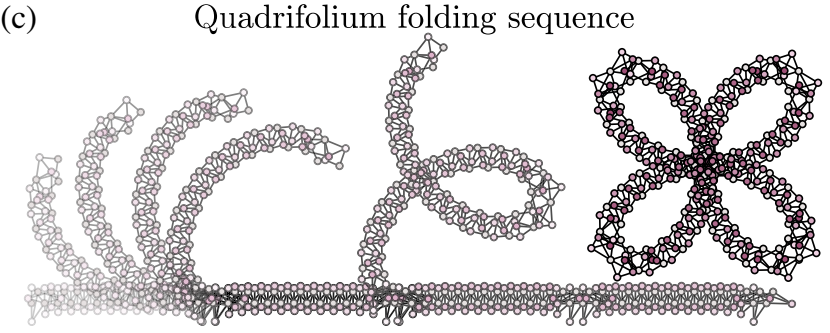

FIG. 9. Designing precise network geometry. (a) To design a network chain that forms a desired curve, we tessellate the curve with isosceles triangles, where each triangle represents a unit. The gold edges represent $l_{k}$ and $l_{k+1}$, while the purple edges represent $c$. (b) The units are then constructed by placing extra nodes along the solution space defined by the start and end positions (Sec. IV B). Then, the units are combined into a chain by merging nodes (Sec. IV C). (c) The resulting network consists of nonidentical units that have different bond lengths, and thereby, the network has nonperiodic structure. However, because all units are designed to share the same start fixed point $l^{\bullet}$ and end fixed point $l^{\circ}$, the network starts at a periodic state at $l_{k}=l^{\bullet}$ and undergoes one conformational motion to form the desired curve at the second periodic state $l_{k}=l^{\circ}$.

Now that we have the desired end coordinates for nodes 1,2 , and 3 of each unit, we construct each unit according to Sec. IV B by placing two nodes $j \in\{4,5\}$ satisfying
Eq. (16), and we combine units precisely according to Sec. IV C by merging the nodes corresponding to the shared corners between neighboring triangles to form a network [Fig. 9(b)]. At the start fixed point $l_{k}=l^{\bullet}$, the network begins as a line because all lengths $c=l^{\bullet}$. At the end fixed point $l_{k}=l^{\circ}$, the shape variables reach their programmed length $c=c^{\circ}$, thereby forming the quadrifolium [Fig. 9(c)].

\section{F. Summary of network shape design}

In summary, we successfully achieved our first goal, to understand the organizational principles that enable us to design precise shape changes [Fig. 2(b)] by designing units with one conformational motion that transition between two fixed points with tunable shape (Sec. IV B), and by choosing and combining nonidentical units whose end shapes trace out the desired shape (Sec. IV E).

\section{DESIGNING THE CONFORMATIONAL SEQUENCE USING STABILITY}

Now that we have the principles for constructing complex shape changes, we move on to our second aim, to design a network's folding sequence by designing the stability of the maps of its component units [Fig. 2(c)]. Recall from Sec. III that the folding sequence of a network depends on the stability of the maps of the component units. If all units are in a conformation that is stable (i.e., $\left|\mathrm{d} l_{k+1} / \mathrm{d} l_{k}\right|<1$ ), then a perturbation at $l_{1}$ decays across units, and the network begins folding at the $l_{1}$ end [Fig. 5(d)]. Alternatively, if all units are in a conformation that is unstable, then a perturbation at $l_{1}$ grows across units, and the network begins folding at the $l_{k+1}$ end [Fig. 5(b)]. How can we tune the stability of our units' maps to design the folding sequence?

\section{A. Motivating stability design}

To design a unit's stability, we must first keep in mind that there already exist constraints from designing a unit's shape in Sec. IV. In Sec. IV B, we already designed our unit comprising three nodes, $i \in\{1,2,3\}$, to successfully transition from desired start positions $\left(x_{i}^{\bullet}, y_{i}^{\bullet}\right)$ to the desired end positions $\left(x_{i}^{\circ}, y_{i}^{\circ}\right)$ along one conformational motion (Fig. 6). To do this, we added two additional nodes $j \in$ $\{4,5\}$ and fully connected them to the first three nodes for a total of six edges $\mathcal{E}=\{1,2,3\} \times\{4,5\}$ to yield one conformational motion [Fig. 6(c)].

We found that these additional nodes could not be placed arbitrarily in space. Once we fix the start positions of the added nodes, $\left(x_{j}^{\bullet}, y_{j}^{\bullet}\right)$, we also fix the edge lengths, and most choices of edge lengths cannot remain constant at the start and end positions according to Eq. (16). Instead, in Sec. IV B, we found that the start positions of each of the added nodes must lie on a one-dimensional conic section, 
(a) Find solution space $\begin{array}{ll}\text { O Start } & \text { o o Added } \\ \circ \text { End } & \text { Solution }\end{array}$

Add nodes at $\theta_{4}$ and $\theta_{5}$

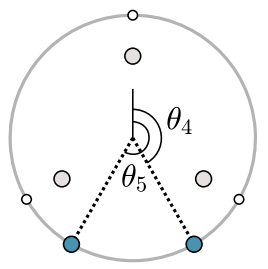

(b) Construct network slope $=d l_{2} / d l_{1}$

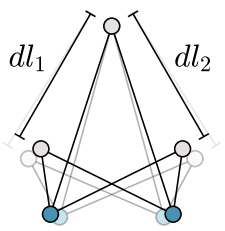

(c) Calculate slope at each added node position: $\left|d l_{2} / d l_{1}\right|$

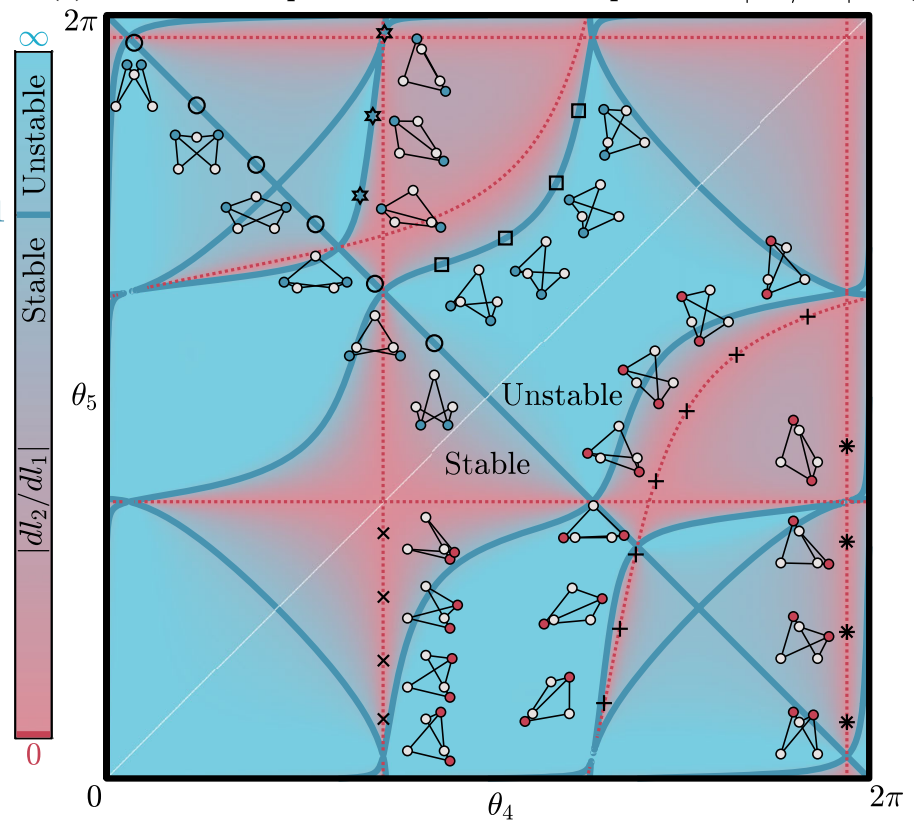

(d) Metastable $\left|d l_{2} / d l_{1}\right|=1$

(e) Superstable oSymmetric 1 $\left|d l_{2} / d l_{1}\right|=0$

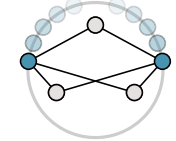

o Symmetric 2

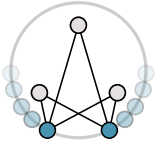

口Asymmetric 1

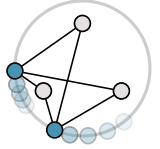

Asymmetric 2 +2 colinear

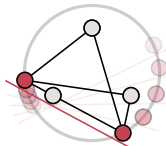

+2 colinear

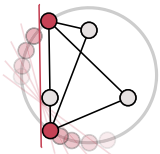

$\times 1$ colinear
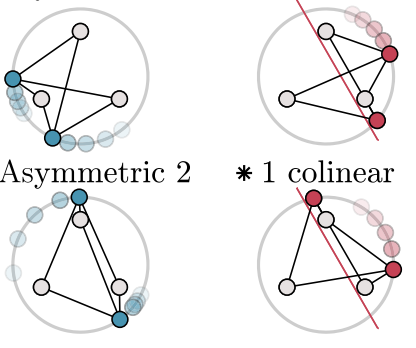

FIG. 10. Designing the sequence of conformational change. (a) Schematic of the addition of two nodes along the solution space, parametrized by angles $\theta_{4}$ and $\theta_{5}$. (b) At the start position, connecting the added nodes to the initial nodes with edges yields one conformational motion, characterized by a slope of $\mathrm{d} l_{2} / \mathrm{d} l_{1}$. (c) Phase diagram of the slope magnitude at all placements of added nodes. The solid blue line marks the transition between stable and unstable. The red dashed line marks where the slope equals zero. (d) Units with $\mid$ slope $\mid=1$ and (e) with $\mid$ slope $\mid=0$.

the solution space, for the subsequently fixed edge lengths to remain rigid at the start and end positions (Fig. 7).

An immediate question that arises is precisely where on the solution space should nodes $j \in\{4,5\}$ be placed? If each node $j$ can be placed anywhere along the onedimensional solution space, then for the two added nodes, we are left with a two-dimensional parameter space (onedimensional solution space per node) along which we can add nodes $j \in\{4,5\}$. In addition to the unit shape, can we use these two dimensions to design the stability of the unit at these shapes?

\section{B. Searching the parameter space}

The positions of the added nodes determine the stability of a unit, which in turn determines the sequence of the full network's shape change. To design a unit's stability, we establish general principles of node placement through the detailed study of one unit whose initial and final node positions generate a circular solution space, along which we add node 4 at $\theta_{4}$ and node 5 at $\theta_{5}$ [Fig. 10(a)]. At each position $0 \leq \theta_{4}, \theta_{5}<2 \pi$, we connect the added nodes $j \in$ $\{4,5\}$ to all of the original nodes $i \in\{1,2,3\}$ and compute the slope of length changes as a function of the node positions [Figs. 10(b) and 10(c)].

We observe consistent patterns of node positions for units that are maximally stable (superstable [42]), where $\left|\mathrm{d} l_{2} / \mathrm{d} l_{1}\right|=0$. Similar to a stable configuration where a perturbation in length $l_{1}$ decays as it propagates to length $l_{2}$, a superstable configuration means that a perturbation in length $l_{1}$ yields no response to linear order in length $l_{2}$. Hence, a network comprising units in superstable configurations will fully localize their shape change at the $l_{1}$ end to linear order. We observe that superstable units entail that both added nodes are colinear with the node $i=1$ that exclusively defines $l_{1}$, or that at least one added node is colinear with both nodes $i=2,3$ defining $l_{2}$ [Fig. 10(e)]. The former condition guarantees that the sole motion of node 1 (perpendicular to the colinearity) is a conformational motion, such that $\left|d l_{1}\right| \geq 0$ while $\left|d l_{2}\right|=0$. The latter condition guarantees that the motion of nodes 2 and 3 is perpendicular to the direction of their length, such that $\left|d l_{2}\right|=0$ while $\left|d l_{1}\right| \geq 0$. Hence, we ensure stable units in the quadrifolium by placing nodes near the first colinear condition (Fig. 9).

We also observe consistent patterns of node positions for units at the transition between stable and unstable (marginally stable) where $\left|d l_{2} / d l_{1}\right|=1$ [Fig. 10(d)]. Marginally stable units encompass all symmetric node positions $\theta_{4}=-\theta_{5}$, whereby the positions of the added nodes are mirrored across the vertical axis. Additionally, marginally stable units consist of more complex asymmetric node positions [Fig. 10(d)].

\section{Summary of stability design}

In summary, we successfully achieve our goal of designing not only the shape [Fig. 2(b)] but also the folding 
sequence [Fig. 2(c)] of a network chain by designing the stability of its component units. We find that stable units can be designed through the colinear placement of added nodes 4 and 5 [Fig. 10(e)], while marginally stable units can be designed through the symmetric placement of added nodes [Fig. 10(d)]. Importantly, these principles generalize to solution spaces that are not circles, and we used these principles to choose the stability of the units in Fig. 7 and in the quadrifolium Fig. 9.

\section{SUPERSTABILITY AND THE MECHANICAL AND GATE}

\section{A. Motivating superstable convergence}

Now that we have achieved the design of network shape and folding sequence [Figs. 2(b) and 2(c), we move on to the discovery of exotic and nonlinear behavior [Fig. 2(d)]. We have already encountered one such inherently nonlinear phenomenon through superstable unit conformations [Fig. 10(e)], where a change in length $l_{1}$ yields no change in length $l_{2}$ to linear order. This means that a network comprising units at a superstable fixed point will converge to that fixed point at a faster-than-linear rate. What implications does superstability have for our ability to design networks with nonlinear behavior?

\section{B. Utilizing superstable convergence}

Networks at a superstable fixed point demonstrate a qualitatively more extreme localization of shape change than those at merely stable fixed points. To formalize this concept, we take the second-order Taylor series expansion of a unit's map, $l_{k+1}=f\left(l_{k}\right)$, about a fixed point $l^{*}$ with slope $s=f^{\prime}\left(l^{*}\right)$ and half of the curvature $t=f^{\prime \prime}\left(l^{*}\right) / 2$ to yield

$$
\Delta l_{k+1} \approx s \Delta l_{k}+t \Delta l_{k}^{2}
$$

A network at a stable fixed point (e.g., $s=0.1$ ) converges linearly because the quadratic term $\Delta l_{k}^{2} \ll \Delta l_{k}$ becomes negligibly small, such that an infinitesimal perturbation $d l_{1}$ cannot be registered to numerical precision $\left(10^{-16}\right)$ after unit $k=16$. In contrast, a network at a superstable fixed point [ $s=0$, Figs. 11(a) and 11(b)] converges quadratically because the linear term vanishes, such that an infinitesimal perturbation $d l_{1}$ cannot be registered in unit $k=2$. Even a finite displacement $\Delta l_{1}=0.1$ propagates to a $\Delta l_{k+1}$ that is smaller than $10^{-16}$ after unit $k=4,10^{-32}$ after unit $k=5$, and $10^{-64}$ after unit $k=6$ [Fig. 11(c)]. As a reference, the ratio of diameters between a classical electron and the observable universe is around $10^{-42}$.

This severe localization of shape change renders the $l_{k+1}$ end effectively rigid, thereby allowing us to design networks with unexpected and nonlinear functions. Here, we demonstrate a mechanical instantiation of an AND gate, which is a binary operator with one Boolean output that depends on two independent Boolean inputs. The Boolean states are the unit's two fixed points, $l^{\circ}=\sqrt{3}$ and $l^{\circ}=3$ [Fig. 11(a)], and each of the gate's inputs is the length $l_{1}$ of a network [Fig. 11(b)]. We combine the two networks by merging the indicated nodes at and near length $l_{11}$ to form our mechanical AND gate [Figs. 11(d)-11(i), see Sec. XI of Ref. [40] for a physical network).

By constraint counting from Eq. (2), the AND gate should have one finitely deformable conformational motion, even if it exists at a kinematic bifurcation allowing for two infinitesimal motions [21]. However, because of the quadratic convergence to the fixed point $l^{\circ}$, the AND gate begins (a) Unit conformational motion

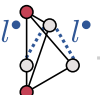<smiles>C1OC2OC1O2</smiles>

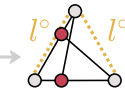

(b) Network of combined units

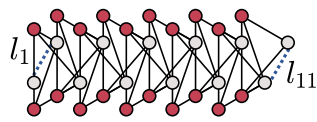

(c) Quadratic convergence to $l^{\bullet}$

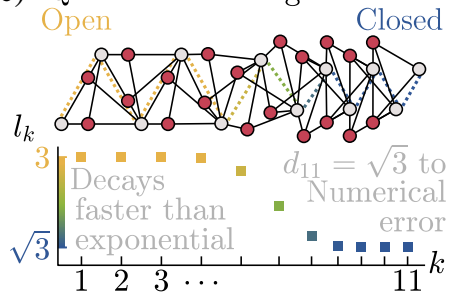

(d) Mechanical AND gate

Inputs
$l_{\mathrm{a}}, l_{\mathrm{b}}$
Output
$l_{\mathrm{o}}$

Join 2 networks

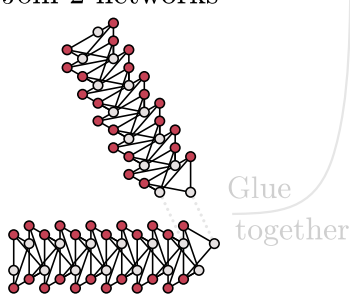

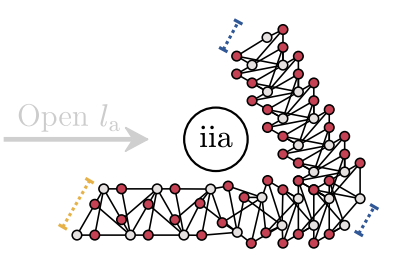
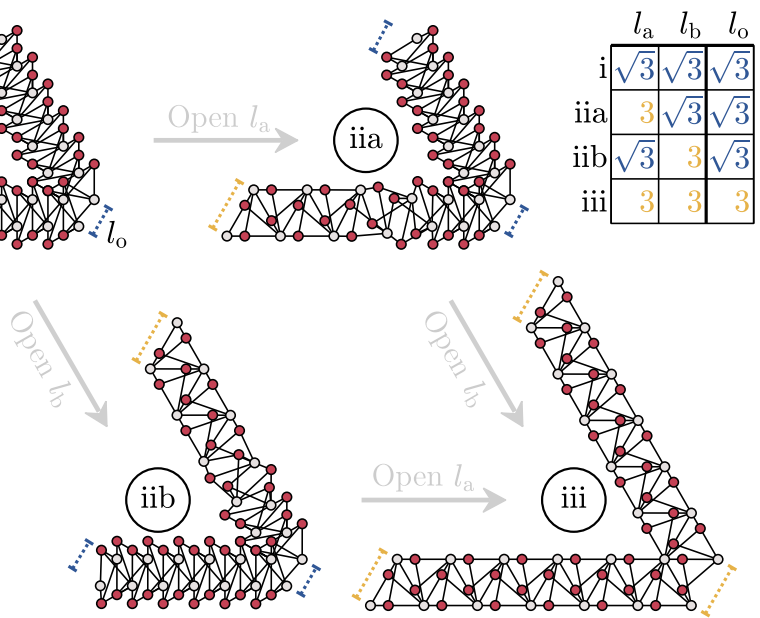

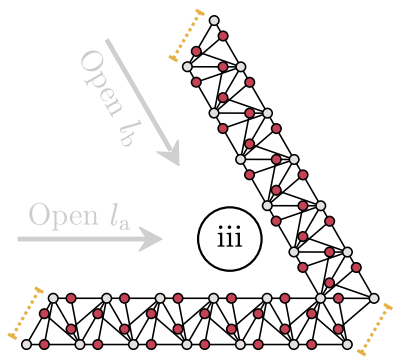

FIG. 11. Superstability and extreme localization through faster-than-exponential convergence. (a) Conformational motion of a unit from the superstable fixed point $l^{\bullet}$ to the unstable fixed point $l^{\circ}$. (b) Copies of this unit combine to form a network chain that (c) converges to $l^{\bullet}$ at a faster-than-exponential rate. (d) Two of these network chains, $a$ and $b$, combine to form a mechanical AND gate, where the inputs $l_{a}$ and $l_{b}$ are the $l_{1}$ ends of each network. From $i$, the gate has two independently deformable conformational motions to form any linear combination of iia and iib. The signal can only continue to $l_{o}$ when both inputs are open at $l_{a}=l_{b}=3$. 
at a geometry that effectively has two independent and finite motions [Fig. 11(d-iia) and 11(d-iib)] and ends at a geometry that only has one such motion at $l_{11}$ [Fig. 11(d-iii)]. Hence, the AND gate can finitely access all four combinations of Boolean inputs, while only one allows for the propagation of the mechanical signal.

\section{PERIOD DOUBLING ROUTE TO MECHANICAL CHAOS}

\section{A. Motivating chaotic divergence}

At the opposite extreme, networks comprising units that lose their stability undergo divergent shape changes that are unpredictable and chaotic. Until now, every unstable fixed point has been accompanied by a stable one, to which each subsequent unit $k+1$ eventually converged. If there is no accompanying stable fixed point, to where would the units converge?

\section{B. Designing chaotic divergence}

To answer this question, we design the slope of a unit at a fixed point by drawing on prior work [14] to constrain the motion of unconnected nodes $i \in\{1,2,3\}$. We define $l_{1}$ and $l_{2}$ to be the length between node pairs $\{1,2\}$ and $\{2,3\}$, respectively, and place the nodes at $\left(x_{i}, y_{i}\right)$ such that the lengths equal a fixed point $l_{1}=l_{2}=1$ [Fig. 12(a)]. We then choose the instantaneous node motions $\left(d x_{i}, d y_{i}\right)$ to achieve a desired slope $s=d l_{2} / d l_{1}$, where we fix $d l_{1}=1$ as constant, and vary $d l_{2}$. To achieve $\left(d x_{i}, d y_{i}\right)$ as the sole conformational motion, we connect all nodes $i$ to an added node $j$ and solve for the positions $\left(x_{j}, y_{j}\right)$ and motions $\left(d x_{j}, d y_{j}\right)$ that keep all edge lengths constant by satisfying the derivative of Eq. (1):

$$
\left(x_{i}-x_{j}\right)\left(\mathrm{d} x_{i}-\mathrm{d} x_{j}\right)+\left(y_{i}-y_{j}\right)\left(\mathrm{d} y_{i}-\mathrm{d} y_{j}\right)=0 .
$$

We call these node positions $\left(x_{j}, y_{j}\right)$ the solution space, along which we add two nodes $j \in\{4,5\}$ to yield a network with one conformational motion that achieves the designed slope at the fixed point [Fig. 12(b), see Sec. I of Ref. [40] for the design algorithm, Sec. III for the analytical form of the iterated map, Sec. IV for conditions for a unit conformational motion to act as a map, and Ref. [14] for additional details].

For a unit designed with a stable fixed point $(|s|<1)$, subsequent units converge to the fixed point and assume the same shape [Fig. 12(b)]. As we design units with more negative slopes $s<-1$, the fixed point undergoes a bifurcation and becomes unstable, giving birth to a stable 2-cycle where every second unit in the network repeats (a) Design motion

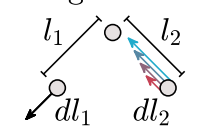

Solution space

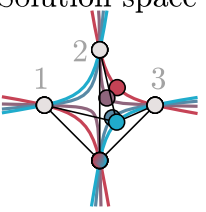

(b) From stable to unstable
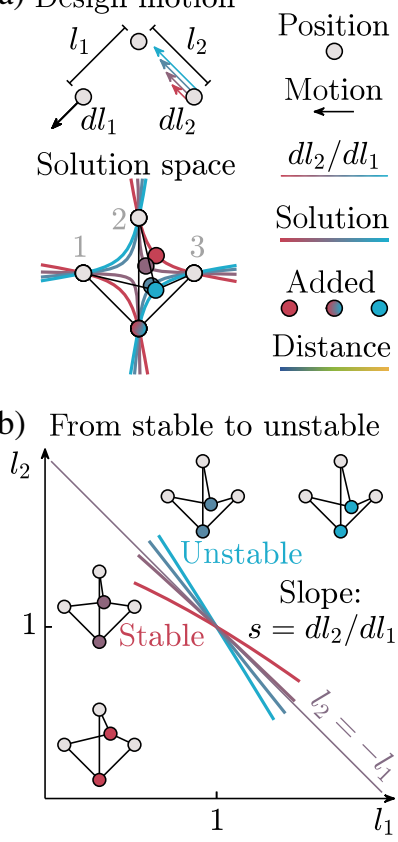

Solution

Added

Distance (c)
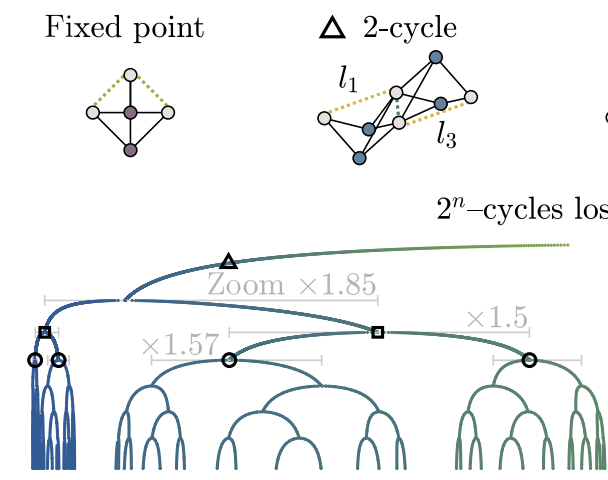

(d) Chaotic modules yield non repeating lattice

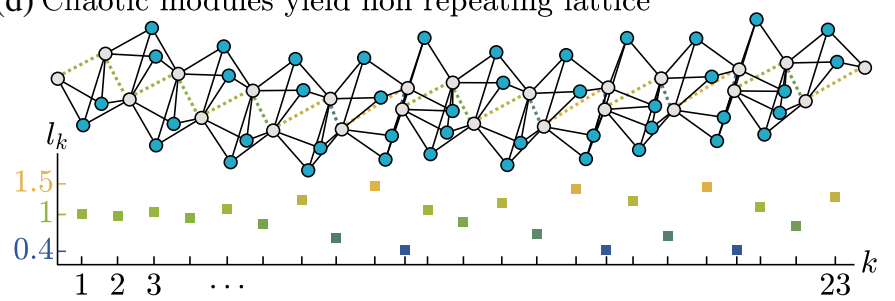

Period doubling route to chaos
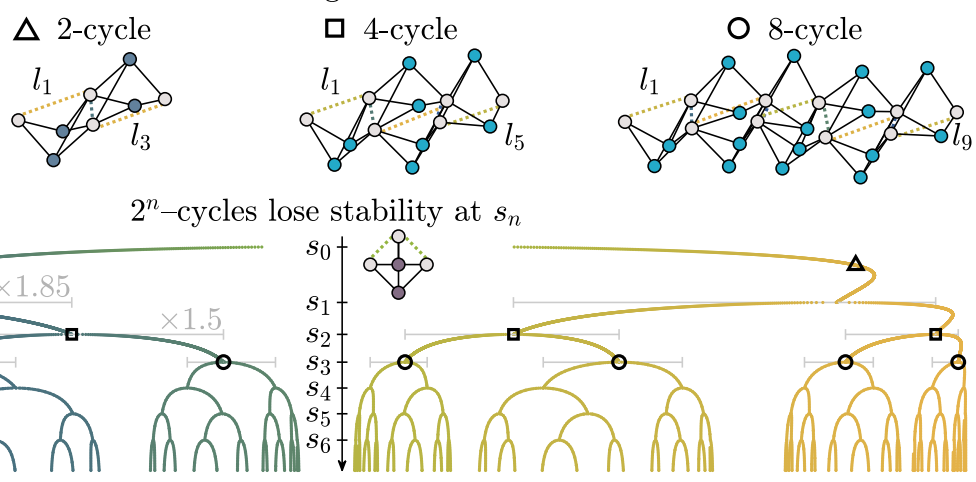

(e) Feigenbaum constant

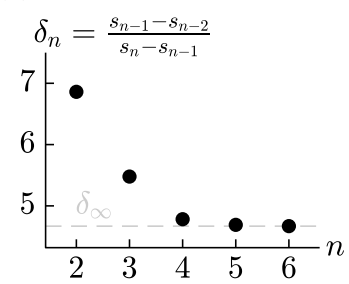

FIG. 12. Mechanical chaos. (a) Schematic of the stability design process at the fixed point $l_{1}=l_{2}=1$, with the designed instantaneous node motions in black and colored arrows, the corresponding solution spaces in colored lines, and the positions of the added nodes for each unit in colored nodes. (b) Slope plots of the conformational motion for each unit. (c) At a slope of $s_{0}=-1$ (left), the fixed point $l^{*}=1$ is marginally stable. As the slope passes $s_{1}$, the fixed point becomes unstable, and a new stable 2-cycle is born (triangle). As the slope continues past $s_{n}$, each $2^{n}$-cycle becomes unstable and gives birth to a stable $2^{n+1}$-cycle, until (d) the unit becomes chaotic with no stable cycles. (e) The ratio of slopes at the bifurcations converges to the Feigenbaum constant. The slope values in this example are $\left(s_{0}, s_{1}, s_{2}, s_{3}, s_{4}, s_{5}, s_{6}, s_{7}\right) \approx(-1,-1.522545,-1.574527,-1.582104,-1.583487,-1.583777,-1.583838$, $-1.583851)$. 
(Fig. 12, triangle). As we design units with increasingly negative slopes, the 2-cycle becomes unstable at slope $s=s_{1}$, giving birth to a stable 4-cycle (Fig. 12, square), which then becomes unstable at $s=s_{2}$ and gives birth to a stable 8-cycle (Fig. 12, circle). Continuing this process, each $2^{n}$-cycle loses stability at $s_{n}$ and gives birth to a stable $2^{n+1}$-cycle, until the network loses all stable cycles and becomes chaotic [Fig. 12(d)]. This process is known as a period-doubling bifurcation and is characterized by the Feigenbaum constant [43]

$$
\delta_{\infty}=\lim _{n \rightarrow \infty} \frac{s_{n-1}-s_{n-2}}{s_{n}-s_{n-1}} \approx 4.669,
$$

to which our units converge [Fig. 12(e)]. Additionally, the chaotic evolution of our units is captured by the Lyapunov exponent that quantifies the rate of divergence of subsequent units from infinitesimally nearby initial units, and it is given by

$$
\lambda\left(l_{1}\right)=\lim _{n \rightarrow \infty} \frac{1}{n} \sum_{k=1}^{n} \ln \left|f^{\prime}\left(l_{k}\right)\right|
$$

We estimate the exponent by averaging across long trajectories from many initial conditions to be 0.246 (see Sec. V of Ref. [40] for the calculation of the exponent), demonstrating positive divergence: a hallmark of chaos.

\section{PERIOD THREE IMPLIES MECHANICAL CHAOS}

\section{A. Motivating 3-cycle units}

In the previous section, as we changed the edge lengths of a unit to lose stability at a fixed point, the unit underwent a period-doubling route to chaos. While the presence of many-period cycles may be useful for designing metamaterial lattices, each choice of edge length only corresponded to a specific $2^{n}$-cycle. Can a single superunit with fixed edge lengths yield arbitrarily many cycles?

\section{B. A 3-cycle unit and Sharkovsii's theorem}

To obtain such a superunit, all we require is for the unit's map to display a 3-cycle. This requirement is a direct result of Sharkovsii's theorem, which states that for any real interval $I \subset R$, if a map $f: I \rightarrow I$ has a point of period 3, then it contains a point of period $k$ where $k$ is a positive integer [44]. This deceptively simple statement leads to powerful consequences, as a unit whose map contains a 3cycle not only implies chaos [45], but it also implies that it can change its shape to yield any integer-period cycle.

We discover such a 3-cycle unit [Fig. 13(a)] and also demonstrate the presence of other positive-integer cycles such as 2-cycles and 6-cycles [Fig. 13(b)]. Importantly, unlike the unit in the period-doubling route to chaos, this (a) Cobweb plot of a unit that contains a 3-cycle

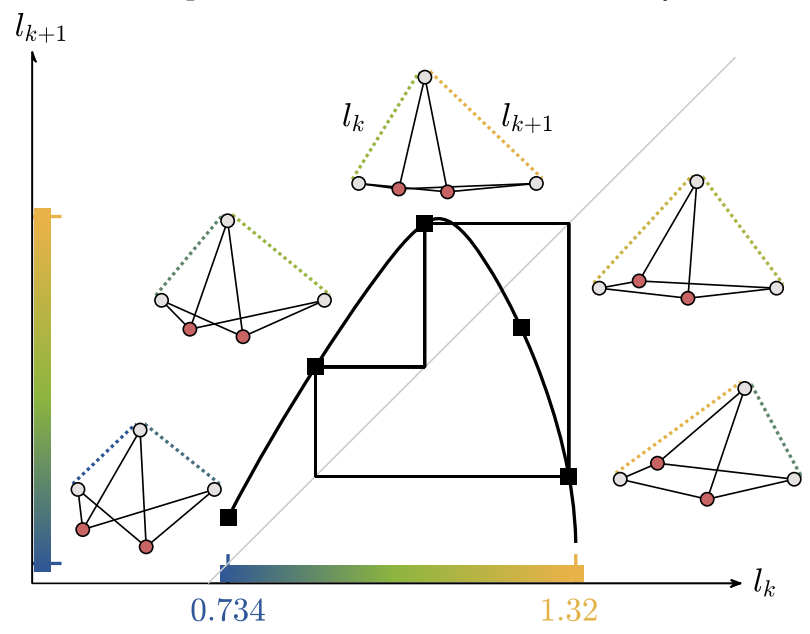

(b) Examples of 2-cycle, 3-cycle, and 6-cycle
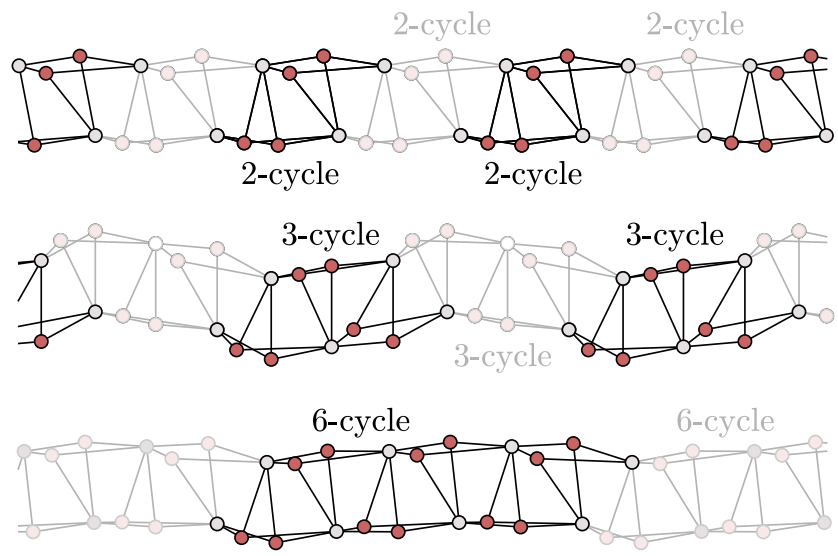

FIG. 13. A 3-cycle unit. (a) Cobweb plot of a unit containing a 3-cycle. (b) Examples of 2-cycle, 3-cycle, and 6-cycle conformations that can be found in this unit.

unit contains cycles of all positive integer periods with one single set of edge weights.

\section{CONSTRUCTING PHYSICAL NETWORKS}

Here, we implement this theory for designing the geometry of both the sequence and macroscopic structure of mechanical networks by constructing physical networks. We construct a superstable and sequentially collapsible network by laser cutting the edges from $1 / 8$-inch thick acrylic and connecting their joints using Chicago screws [Figs. 14(a) and 14(b)]. Additionally, many deployable applications [46] require a compact initial geometry and a precise, rigid final geometry. Using wooden sticks that are joined by a staple prong at the joints, we show a four-bar linkage with two fixed points $l^{\circ}$ and $l^{\circ}$, where the $l^{\circ}$ point is superstable [Fig. 14(d)]. These modules combine in a chain [Fig. 14(e)] that yields a wide spiral with an open channel at $l^{\circ}$ and collapses to a narrow spiral with no channel at $l^{\circ}$ 
(a) Laser cut acrylic: Superstable

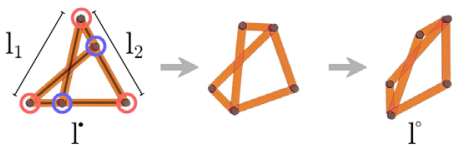

(b)
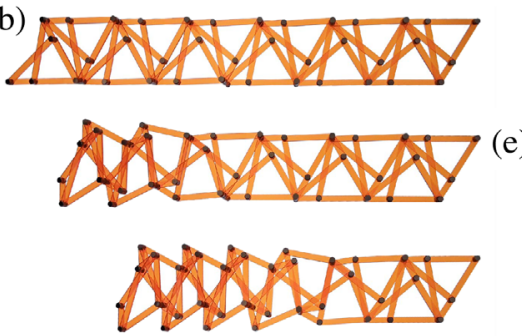

$\stackrel{\longmapsto}{10 \mathrm{~cm}}$

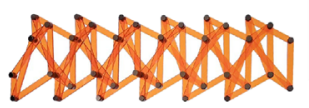

(c) Wood: Deployable

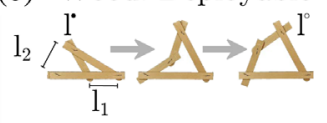

(d)

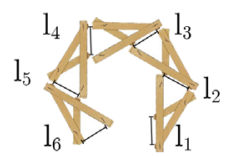

(e)

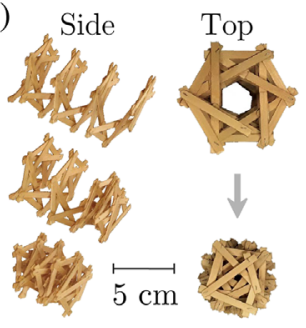

(f) Origami: Deployable

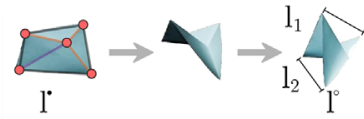

(g) Modules

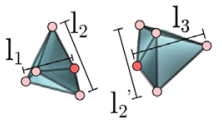

Combine (j)

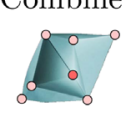

(h)

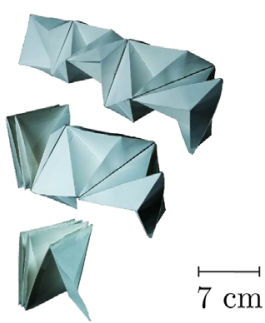

(i) 3D printed flexible: Planar

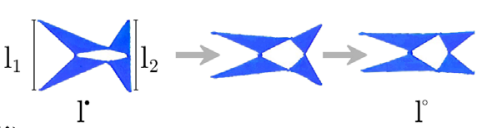

(j)

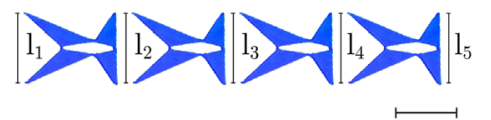

(k)
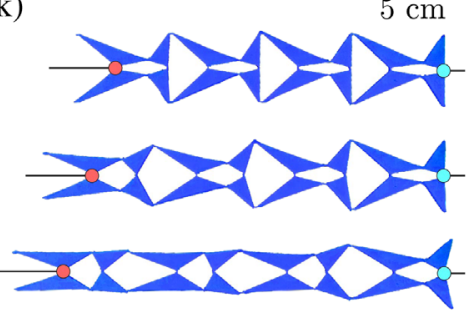

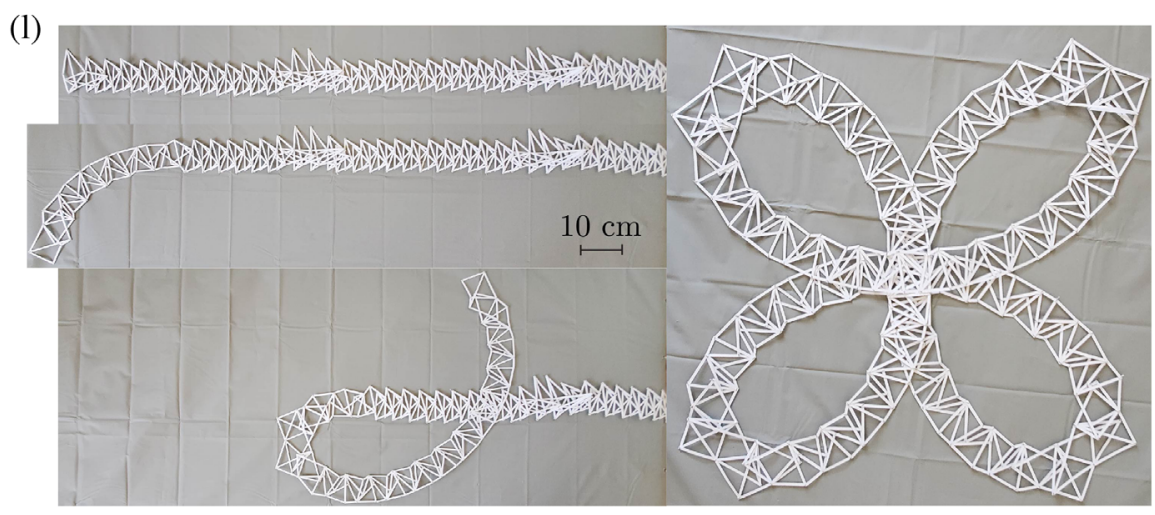

(m)

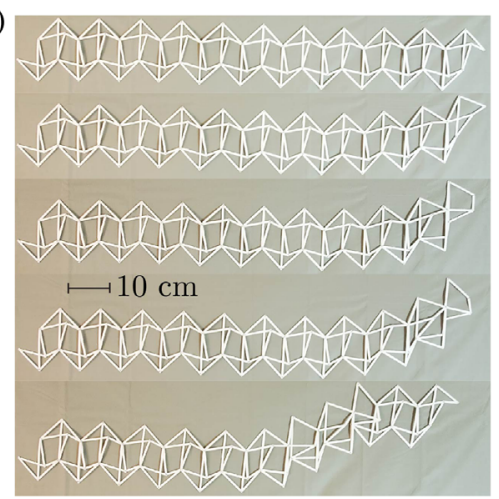

FIG. 14. Physical construction of networks. (a) Photo of a superstable unit constructed from laser-cut acrylic bars held together by Chicago screws at the joints, transitioning between two fixed points $l^{\bullet}$ and $l^{\circ}$. (b) Photos of a combined network collapsing from $l^{\bullet}$ to $l^{\circ}$. (c) A four-bar linkage with two fixed points $l^{\bullet}$ and $l^{\circ}$, (d) combined hexagonally into (e) an initially wide spiral helix with a channel $l^{\bullet}$, collapsing sequentially to a narrow closed helix. (f) Photo of a creased square sheet of paper modeled as a linkage with one conformational motion moving between two fixed points $l^{\bullet}$ and $l^{\circ}$ (purple is the mountain fold; orange is the valley fold). (g) Two creased sheets combined by merging the nodes defining $l_{2}$ and $l_{2}^{\prime}$, along with a third node in each module marked in bright red. (h) Combined network of 12 sheets that sequentially collapses from the $l^{\circ}$ to the $l^{\bullet}$ lattice. (i) A 3D-printed planar module with two fixed points $l^{\bullet}, l^{\circ}$. Each module is composed of triangles connected by a thin layer of material, which (j) form a chain where (k) fixing the cyan hinge and pulling the red hinge yields a sequential transition from $l^{\bullet}$ to $l^{\circ}$. (1) Photos of the quadrifolium and (m) chaotic networks made from cardstock bars held together by metal pins.

[Fig. 14(f), see Secs. VI and VII of Ref. [40] for the maps $l_{k+1}=f\left(l_{k}\right)$ ].

To demonstrate the generalizability of our framework to three-dimensional space, we model a creased square of paper as a linkage, where each crease is a rigid edge, and the intersection of creases is a node [Fig. 14(f)]. We define $l_{1}$ and $l_{2}$ to be the distances between opposing corners in this sheet, which collapses from the unfolded $l^{*}$ to the folded $l^{\circ}$ crystalline states. We combine these modules by merging the nodes defining $l_{2}$ and $l_{2}^{\prime}$ [Fig. 14(g)] to obtain an origami structure that collapses sequentially to a flat geometry.

These principles also extend to planar networks comprised of polygons (e.g., triangles) connected at vertices through a thin layer of flexible material [Fig. 14(g)]. We design a module with two fixed points $l^{\circ}$ and $l^{\circ}$, where the initial point $l^{\bullet}$ is superstable. We can chain these modules as before to yield the same iterated map $l_{k+1}=f\left(l_{k}\right)$ [Fig. 14(h)], such that we obtain a sequential transition from $l^{\circ}$ to $l^{\circ}$ by pulling on the network [Fig. 14(i)]. Importantly, because this network is printed as shown, there is no required assembly (see Sec. X of Ref. [40] for the bond lengths of networks and Section XI for considerations of elastic bond lengths).

\section{ELASTICITY AND SIGNAL PROPAGATION IN THE MECHANICAL AND GATE}

Until now, we have assumed that the bonds were rigid or that any elastic deformations would decay to an equilibrium configuration of zero extension. However, real systems can sustain deformations from resistance to motion such as 
friction, which may alter the designed behavior of these networks. Here, we provide two experiments to quantify the effect of bond deformations.

In the first experiment, we quantify the propagation of the input signal through a chain of superstable units with elastic bonds. We take the superstable unit shown in the bottom-left part of Fig. 15(a) and replace the rigid bonds with elastic bonds of unit stiffness. Then, we force the input $l_{1}$ open to 3 , force the output $l_{2}$ to $\sqrt{3}$, and compute the maximum bond strain at the equilibrium configuration. We repeat this procedure for longer chains comprising a range from 2 to 8 units and plot the max strain [Fig. 15(a)]. There is an appreciable strain up to around 5-6 units. Hence, enough of the input signal propagates to the output to appreciably deform the bonds. If the output is not fixed at $\sqrt{3}$, then the bonds must deform appreciably for the input signal to fully decay.

Importantly, this first result holds true for any scalar multiple of bond stiffness. Specifically, the bonds are modeled as linear springs with potential energy given by

$$
V=\frac{1}{2} \sum_{(i, j) \in \mathcal{E}} k_{i j}\left(l_{i j}^{*}-l_{i j}\right)^{2}
$$

(a) Percent strain when fixing input to 3 and output to $\sqrt{3}$

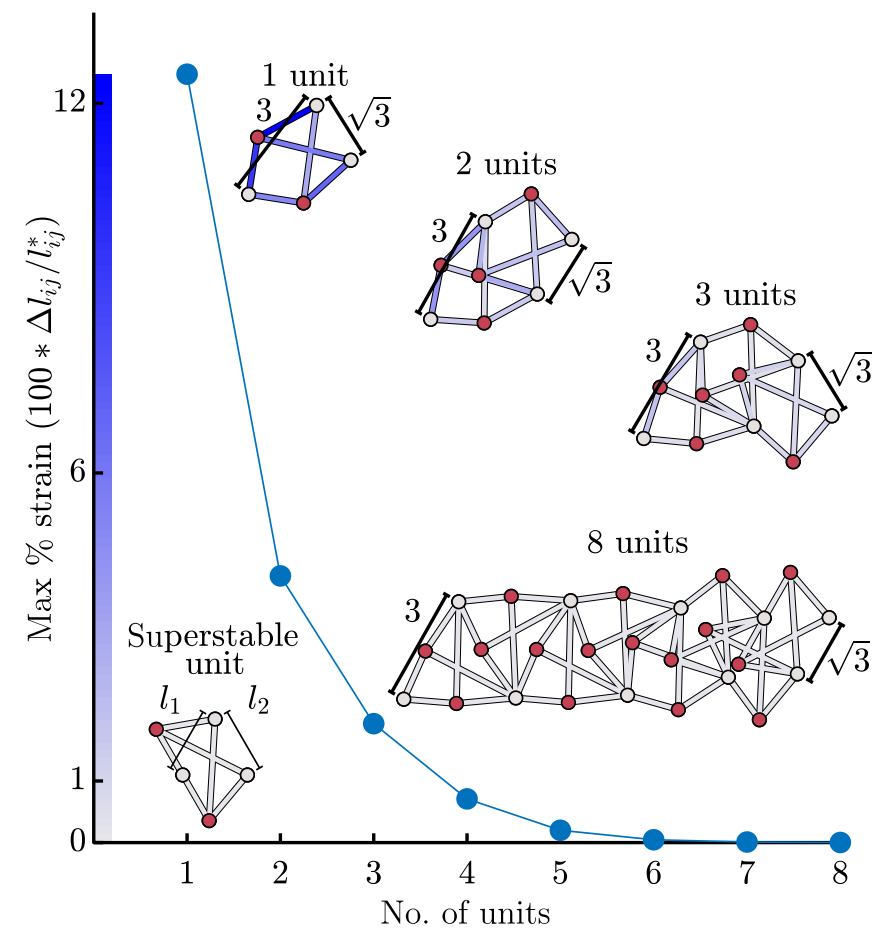

where $\mathcal{E}$ is the set of all bonds in the network, $k_{i j}$ is the stiffness of the bond connecting node $i$ to node $j, l_{i j}^{*}$ is the equilibrium length of said bond, and $l_{i j}$ is the current length of said bond. Because potential energy is linear in bond stiffness, the location of the minimum potential configuration and the percent bond strain do not change with scalar multiples of $k_{i j}$.

In the second experiment, we construct AND gates by combining chains of elastic superstable units [Fig. 15(b), top] and measure both the energetics and geometry of these AND gates. To directly quantify these effects, we construct AND gates by combining chains of one unit, two units, three units, and four units [Fig. 15(b)]. We systematically fix the distance between the input nodes, $l_{a}$ and $l_{b}$, across a range from $\sqrt{3}$ to 3 , and evolve the node coordinates to minimize the potential energy with all $k_{i j}=1$. Then, we plot the distance between the output nodes, $l_{o}$, along with the maximum percent strain [Fig. 15(b)].

We find that an AND gate comprising one unit has the greatest signal propagation with a large percent strain, but it also has nonideal geometric behavior. Specifically, the output fails to remain fully closed when only one of the two inputs is open [Fig. 15(b)]. However, as we increase (b) Max length change when fixing $l_{a}$ and $l_{b}$ but not $l_{o}$

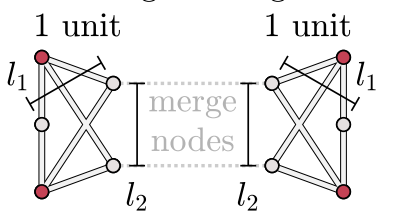

AND gate with 1 unit

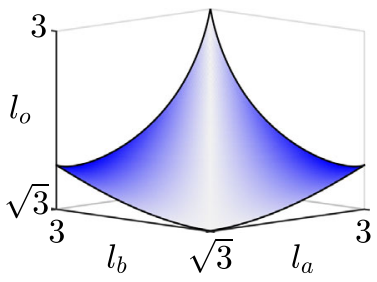

AND gate with 3 units

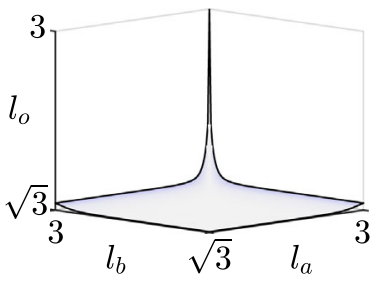

AND gate with 2 units

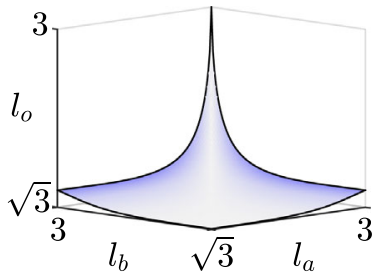

AND gate with 1 units

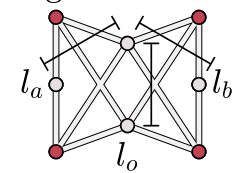

AND gate with 4 units

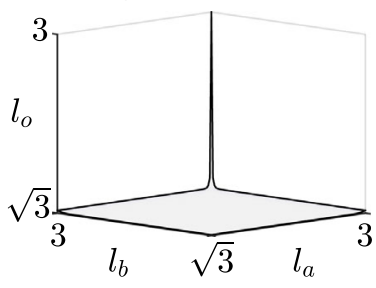

FIG. 15. Elastic deformations in superstable networks. (a) Plot of the maximum percent strain of the elastic bonds in networks comprising $k$ superstable units. The initial length $l_{1}=3$ is fixed to be open, the final length $l_{k+1}=\sqrt{3}$ is fixed to be closed, and the minimum energy configuration is computed for $k=\{1,2, \ldots, 8\}$. (b) Nodes defining length $l_{k+1}$ between two elastic network chains are merged to form an AND gate with input lengths $l_{a}$ and $l_{b}$, and output length $l_{o}$. For $k=\{1,2,3,4\}$, the input lengths are fixed at various values between $\sqrt{3} \leq l_{a}, l_{b} \leq 3$, and are plotted against the output length $l_{o}$ and the maximum percent strain at the minimum energy configuration. 
the number of units in a chain up to 4, we observe that the geometric changes much more closely resemble an ideal AND gate, where the output only opens appreciably when both $l_{a}=l_{b}=3$ are open.

Importantly, we note that in the failure mode of the AND gate-when the inputs $l_{a}=l_{b}=3$ are open but the output $l_{o}=\sqrt{3}$ is forced closed-the maximum percent strain of each branch is identical to that found in Fig. 15(a). This is because the branches are only coupled at the nodes defining $l_{o}$. Hence, the failure mode of the AND gate requires twice as much energy as the failure mode in each branch, while correct operation-when both the inputs and outputs $l_{a}=$ $l_{b}=l_{o}=3$ are open-requires none.

\section{DISCUSSION}

Ever-arising mechanical challenges $[46,47]$ drive the development of innovative designs [31,48-50], which in turn spark novel applications [51,52]. In this work, we presented a simple theory for the principled design of a rich and complex set of folding sequences and large-scale geometries through the properties of a single module. Because of the practical and ubiquitous nature of linkages, these ideas are well-positioned to provide simple solutions to complex problems in robotic grasping [53], deployable mechanisms [46], morphing mechanical structures [47], and tunable metamaterials [54]. By writing the large, nonlinear geometric conformation of a network as the iteration of one module, we retained the richness of network motion while dramatically reducing design complexity.

We studied the fundamental behaviors of this richness, which directly arise from iterated maps. Immediate extensions include designing modules with complex maps (more than two fixed points, negative slopes at fixed points, critical slowing, and bifurcations [43]) and developing principles for combining modules with different maps. The theory can also extend beyond iterated maps, where linkages follow a circular path that is not formally a function $\left(d_{2}\right.$ is not uniquely determined by $\left.d_{1}\right)$. For ease of manufacturing, previous work on planar networks [28] motivates the development of a module design framework specific to these systems. Finally, given the design framework for bistable linkages with elastic bonds [14], a promising future direction lies in the design of sensors, adaptive response, and superelasticity seen in shape memory systems $[55,56]$, deployable structures as seen in origami metamaterials and antennas [46,57], and mechanical computation [58]. Hence, this simple theory provides a versatile and unifying framework for designing large sequential conformational changes in mechanical networks.

Finally, we report in the Sec. XIV of Ref. [40] an analysis of the gender and racial makeup of the authors we cited.

There are no data with mandated deposition used in the paper or the Supplemental Material [40]. All analyses and figures were created in MATLAB and can be publicly accessed at Ref. [59] with a test script that will exactly replicate and save all figures in this paper except the construction of physical networks.

\section{ACKNOWLEDGMENTS}

We gratefully acknowledge Melody X. Lim, Lia Papadopoulos, Jennifer Stiso, Harang Ju, and Dr. Erin G. Teich for conversations and comments on the manuscript, and Karol Szymula for help constructing the acrylic networks. J.Z.K. acknowledges support from the NIH T32-EB020087, PD: Felix W. Wehrli, and the National Science Foundation Graduate Research Fellowship No. DGE-1321851. D. S. B. acknowledges support from the John D. and Catherine T. MacArthur Foundation, the ISI Foundation, the Alfred P. Sloan Foundation, a NSF Career Grant No. PHY-1554488, and from the NSF through the University of Pennsylvania Materials Research Science and Engineering Center (MRSEC), Grant No. DMR-1720530.

[1] J. Li, J. Guo, X. Ou, M. Zhang, Y. Li, and Z. Liu, Mechanical Coupling of the Multiple Structural Elements of the Large-Conductance Mechanosensitive Channel During Expansion, Proc. Natl. Acad. Sci. U.S.A. 112, 10726 (2015).

[2] K. Mori and T. Saito, Effects of Stent Structure on Stent Flexibility Measurements, Ann. Biomed. Eng. 33, 733 (2005).

[3] S. N. Patek, B. N. Nowroozi, J. E. Baio, R. L. Caldwell, and A. P. Summers, Linkage Mechanics and Power Amplification of the Mantis Shrimp's Strike, J. Exp. Biol. 210, 3677 (2007).

[4] C. P. Macol, H. Tsuruta, B. Stec, and E. R. Kantrowitz, Direct Structural Evidence for a Concerted Allosteric Transition in Escherichia coli Aspartate Transcarbamoylase, Nat. Struct. Biol. 8, 423 (2001).

[5] M. Burrows and G. Sutton, Interacting Gears Synchronize Propulsive Leg Movements in a Jumping Insect, Science 341, 1254 (2013).

[6] B. Fu, E. Sperber, and F. Eke, Solar Sail Technology-A State of the Art Review, Prog. Aerosp. Sci. 86, 1 (2016).

[7] L. Zigoneanu, B.-I. Popa, and S. A. Cummer, ThreeDimensional Broadband Omnidirectional Acoustic Ground Cloak, Nat. Mater. 13, 352 (2014).

[8] J. U. Surjadi, L. Gao, H. Du, X. Li, X. Xiong, N. X. Fang, and Y. Lu, Mechanical Metamaterials and Their Engineering Applications, Adv. Eng. Mater. 21, 1800864 (2019).

[9] H. Crapo, Structural Rigidity, Struct. Topol. 1, 26 (1979).

[10] J. C. Maxwell, On the Calculation of the Equilibrium and Stiffness of Frames, Philos. Mag. Ser. 4 27, 294 (1864).

[11] C. R. Calladine, Buckminster Fuller's Tensegrity Structures and Clerk Maxwell's Rules for the Construction of Stiff Frames, Int. J. Solids Struct. 14, 161 (1978).

[12] D. J. Jacobs and M. F. Thorpe, Generic Rigidity Percolation: The Pebble Game, Phys. Rev. Lett. 75, 4051 (1995). 
[13] R. Hartenberg and J. Danavit, Kinematic Synthesis of Linkages (McGraw-Hill Text, New York, 1964).

[14] J. Z. Kim, Z. Lu, S. H. Strogatz, and D. S. Bassett, Conformational Control of Mechanical Networks, Nat. Phys. 15, 714 (2019).

[15] J. M. McCarthy and G. S. Soh, Geometric Design of Linkages (Springer Science \& Business Media, New York, 2010), Vol. 11.

[16] R. Connelly and J.-M. Schlenker, On the Infinitesimal Rigidity of Weakly Convex Polyhedra, Eur. J. Combinatorics 31, 1080 (2010).

[17] M. Stern, C. Arinze, L. Perez, S. E. Palmer, and A. Murugan, Supervised Learning through Physical Changes in a Mechanical System, Proc. Natl. Acad. Sci. U.S.A. 117, 14843 (2020).

[18] J. W. Rocks, H. Ronellenfitsch, A. J. Liu, S. R. Nagel, and E. Katifori, Limits of Multifunctionality in Tunable Networks, Proc. Natl. Acad. Sci. U.S.A. 116, 2506 (2019).

[19] H. Flechsig, Design of Elastic Networks with Evolutionary Optimized Long-Range Communication as Mechanical Models of Allosteric Proteins, Biophys. J. 113, 558 (2017).

[20] C. L. Kane and T. C. Lubensky, Topological Boundary Modes in Isostatic Lattices, Nat. Phys. 10, 39 (2014).

[21] X. Mao and T. C. Lubensky, Maxwell Lattices and Topological Mechanics, Annu. Rev. Condens. Matter Phys. 9, 413 (2018).

[22] K. Sato and R. Tanaka, Solitons in One-Dimensional Mechanical Linkage, Phys. Rev. E 98, 013001 (2018).

[23] D. Z. Rocklin, S. Zhou, K. Sun, and X. Mao, Transformable Topological Mechanical Metamaterials, Nat. Commun. 8, 14201 (2017).

[24] B. Liu, J. L. Silverberg, A. A. Evans, C. D. Santangelo, R. J. Lang, T.C. Hull, and I. Cohen, Topological Kinematics of Origami Metamaterials, Nat. Phys. 14, 811 (2018).

[25] B. G.-G. Chen, B. Liu, A. A. Evans, J. Paulose, I. Cohen, V. Vitelli, and C. D. Santangelo, Topological Mechanics of Origami and Kirigami, Phys. Rev. Lett. 116, 135501 (2016).

[26] D. Melancon, B. Gorissen, C. J. García-Mora, C. Hoberman, and K. Bertoldi, Multistable Inflatable Origami Structures at the Metre Scale, Nature (London) 592, 545 (2021).

[27] A. Rafsanjani, L. Jin, B. Deng, and K. Bertoldi, Propagation of Pop Ups in Kirigami Shells, Proc. Natl. Acad. Sci. U.S.A. 116, 8200 (2019).

[28] C. Coulais, D. Sounas, and A. Alù, Static Non-reciprocity in Mechanical Metamaterials, Nature (London) 542, 461 (2017).

[29] L. A. Lubbers and M. van Hecke, Excess Floppy Modes and Multibranched Mechanisms in Metamaterials with Symmetries, Phys. Rev. E 100, 021001(R) (2019).

[30] M. Stern, V. Jayaram, and A. Murugan, Shaping the Topology of Folding Pathways in Mechanical Systems, Nat. Commun. 9, 4303 (2018).

[31] S. Pellegrino, Deployable Structures, 1st ed. (SpringerVerlag, Berlin, 2001), Vol. 412.
[32] K. Bertoldi, V. Vitelli, J. Christensen, and M. van Hecke, Flexible Mechanical Metamaterials, Nat. Rev. Mater. 2, 17066 (2017).

[33] H. M. Kolken and A. A. Zadpoor, Auxetic Mechanical Metamaterials, RSC Adv. 7, 5111 (2017).

[34] H. M. Kolken et al., Rationally Designed Meta-Implants: A Combination of Auxetic and Conventional MetaBiomaterials, Mater. Horizons 5, 28 (2018).

[35] J. A. Jackson et al., Field Responsive Mechanical Metamaterials, Sci. Adv. 4, eaau6419 (2018).

[36] J. T. Overvelde, T. A. de Jong, Y. Shevchenko, S. A. Becerra, G. M. Whitesides, J. C. Weaver, C. Hoberman, and K. Bertoldi, A Three-Dimensional Actuated OrigamiInspired Transformable Metamaterial with Multiple Degrees of Freedom, Nat. Commun. 7, 10929 (2016).

[37] H. Cui, R. Hensleigh, D. Yao, D. Maurya, P. Kumar, M. G. Kang, S. Priya, and X. (Rayne) Zheng, Three-Dimensional Printing of Piezoelectric Materials with Designed Anisotropy and Directional Response, Nat. Mater. 18, 234 (2019).

[38] Z. Zhao, X. Kuang, J. Wu, Q. Zhang, G. H. Paulino, H. J. Qi, and D. Fang, 3D Printing of Complex Origami Assemblages for Reconfigurable Structures, Soft Matter 14, 8051 (2018).

[39] T. Feagin, A Tenth-Order Runge-Kutta Method with Error Estimate, in Proceedings of the IAENG Conference on Scientific Computing, Hong Kong (2007), https://uhcl-ir.tdl .org/handle/10657.1/2301?show=full, https://sce.uhcl.edu/ feagin/courses/rk10.pdf.

[40] See Supplemental Material at http://link.aps.org/ supplemental/10.1103/PhysRevX.12.011042 for additional methods and results, along with a citation diversity statement.

[41] Y. Zhou, B. G. -g. Chen, N. Upadhyaya, and V. Vitelli, Kink-Antikink Asymmetry and Impurity Interactions in Topological Mechanical Chains, Phys. Rev. E 95, 022202 (2017).

[42] M. H. Lee, Analytical Study of the Superstable 3-Cycle in the Logistic Map, J. Math. Phys. (N.Y.) 50, 122702 (2009).

[43] S. H. Strogatz, Nonlinear Dynamics and Chaos (CRC Press, Boca Raton, FL, 2018).

[44] A. N. Sharkovsii, Coexistence of Cycles of a Continuous Map of the Line into Itself, Int. J. Bifurcation Chaos Appl. Sci. Eng. 05, 1263 (1995).

[45] T.-Y. Li and J. A. Yorke, Period Three Implies Chaos, in The Theory of Chaotic Attractors (Springer, New York, 2004), pp. 77-84.

[46] L. Puig, A. Barton, and N. Rando, A Review on Large Deployable Structures for Astrophysics Missions, Acta Astronaut. 67, 12 (2010).

[47] A. Sofla, S. Meguid, K. Tan, and W. Yeo, Shape Morphing of Aircraft Wing: Status and Challenges, Mater. Des. 31, 1284 (2010).

[48] J. T. B. Overvelde, J. C. Weaver, C. Hoberman, and K. Bertoldi, Rational Design of Reconfigurable Prismatic Architected Materials, Nature (London) 541, 347 (2017).

[49] G. Wei, Y. Chen, and J. S. Dai, Synthesis, Mobility, and Multifurcation of Deployable Polyhedral Mechanisms with Radially Reciprocating Motion, Journal of mechanical design 136, 091003 (2014). 
[50] K. C. Cheung and N. Gershenfeld, Reversibly Assembled Cellular Composite Materials, Science 341, 1219 (2013).

[51] Z. Yang, F. Gao, X. Shi, X. Lin, Z. Gao, Y. Chong, and B. Zhang, Topological Acoustics, Phys. Rev. Lett. 114, 114301 (2015).

[52] S. A. Cummer, J. Christensen, and A. Alù, Controlling Sound with Acoustic Metamaterials, Nat. Rev. Mater. 1, 16001 (2016).

[53] Y. Zheng and W.-H. Qian, Dynamic Force Distribution in Multifingered Grasping by Decomposition and Positive Combination, IEEE Trans. Robotics 21, 718 (2005).

[54] T.-W. Liu and F. Semperlotti, Tunable Acoustic Valley-Hall Edge States in Reconfigurable Phononic Elastic Waveguides, Phys. Rev. Applied 9, 014001 (2018).
[55] A. Lendlein, H. Jiang, O. Jünger, and R. Langer, LightInduced Shape-Memory Polymers, Nature (London) 434, 879 (2005).

[56] Z. Wei, R. Sandstroröm, and S. Miyazaki, Shape-Memory Materials and Hybrid Composites for Smart Systems: Part I Shape-Memory Materials, J. Mater. Sci. 33, 3743 (1998).

[57] J. L. Silverberg, A. A. Evans, L. McLeod, R. C. Hayward, T. Hull, C. D. Santangelo, and I. Cohen, Using Origami Design Principles to Fold Reprogrammable Mechanical Metamaterials, Science 345, 647 (2014).

[58] T. Chen, M. Pauly, and P. M. Reis, A Reprogrammable Mechanical Metamaterial with Stable Memory, Nature (London) 589, 386 (2021).

[59] https://github.com/jk6294/Mechanical_DS.git. 